\title{
Aportaciones históricas y arqueológicas al estudio del proceso de estandarización en la industria ancorera de las potencias marítimas europeas del siglo XVIII
}

Nicolás C. Ciarlo*

Recibido:

17 de septiembre de 2017

Aceptado:

16 de octubre de 2018

\section{Resumen}

Las anclas fueron un elemento de capital importancia a lo largo de la historia de la navegación. En el siglo XVIII, los barcos de guerra europeos utilizaban varios tipos y dependían de ellas para fondear y realizar otras maniobras. La industria ancorera constituyó en aquel entonces una de las expresiones más complejas de la tecnología de forjado del hierro y, en el marco de un proceso de industrialización creciente, estuvo sujeta a varios cambios. En este artículo examinamos las características de las anclas, en materia de diseño y manufactura, de las principales potencias marítimas. A la luz de las fuentes documentales y materiales disponibles, discutimos aspectos relativos al proceso de estandarización y cambio tecnológico en la industria ancorera. Complementariamente, presentamos algunos lineamientos para el estudio de anclas de procedencia arqueológica.

Palabras clave

Industria ancorera Tecnología de forjado Equipamiento náutico Arqueología marítima Siglo XVIII

Historical and archaeological contributions to the study of the standardization process in the anchor industry of the $18^{\text {th }}$ century European maritime powers

\begin{abstract}
Anchors were an element of major importance throughout the history of navigation. In the 18th century, European warships used various types and relied on them to anchor and perform other maneuvers. The anchor industry was at that time one of the most complex expressions of iron forging technology and, under a process of increasing industrialization, it was subject to several changes. In this article, we examine the characteristics of the anchors of the main Maritime powers in terms of design and manufacturing. In light of the available documentary and material sources, we discuss aspects related to the process of standardization and technological change in the anchor industry. Complementarily, we present some guidelines for the study of anchors of archaeological provenance.
\end{abstract}

Keywords

Anchor industry Forging technology Nautical equipment Maritime archaeology $18^{\text {th }}$ century 


\section{Introducción}

Las anclas constituían uno de los elementos más importantes del equipamiento náutico de los barcos. Permitían aferrar un barco al fondo y realizar otras maniobras, por lo que constituían el elemento de seguridad por antonomasia. El sistema de fondeo de las naves estaba compuesto por una dotación de varias anclas, de las que se disponía según las circunstancias. Estas piezas eran producidas por maestros artesanos en establecimientos especializados. Durante el siglo XVIII, en cada región el diseño general de las anclas sobre todo, el de las británicas - sufrió leves modificaciones. Las diferencias más notables se aprecian al comparar los ejemplares utilizados por las diferentes Armadas europeas. En cambio, en las primeras décadas de la siguiente centuria sobrevendrían cambios importantes, entre estos la introducción de nuevos modelos.

Este artículo versará sobre las anclas de hierro con cepo de madera utilizadas por las principales potencias marítimas de Europa. La presentación consta de las siguientes secciones: 1) generalidades sobre las anclas: nomenclatura y descripción de las partes, 2) las anclas de un barco de guerra: tipos, peso, número, disposición y usos, 3) los modelos europeos: rasgos diagnósticos, dimensiones y proporciones generales, e identificación de piezas y 4) la tecnología de fabricación: etapas del proceso de forja, métodos, materiales y calidad de los productos para el servicio naval. A modo de cierre, y a la luz de la evidencia presentada, discutiremos varios aspectos relativos a la industria de anclas durante el período de interés, con foco en el proceso de estandarización y los cambios en el diseño y la manufactura.

\section{Generalidades sobre las anclas}

\section{Elementos de seguridad}

Las anclas de hierro eran instrumentos que, afirmados al extremo del cable y arrojados al agua, servían para aferrar (fondear o anclar) los barcos en puerto $\mathrm{u}$ otro fondeadero $\mathrm{y}$ asegurarlos así frente a la acción de los vientos (Falconer, 1780: ANCHOR; Moore, 1801: ANCHOR; O'Scanlan, 1831, p. 33). A lo largo de la historia se empleó una amplia variedad de anclas ${ }^{1}$. A diferencia de las anclas de la antigüedad, las modernas estaban diseñadas para aferrarse al fondo y soportar un gran esfuerzo antes de ser arrancadas. El ancla recuperada del naufragio del HMS Royal George (1756-1782) llevaba inscripta la leyenda Fear not; I will hold you fast ${ }^{2}$.

2. "No temas; yo te mantendré bien afirmado".

3. La plataforma online del Big Anchor Project de la Nautical Archaeology Society (www. biganchorproject.com) contiene un registro detallado de numerosas piezas localizadas en diversas regiones del mundo. Hasta la fecha, la mayor parte de los registros volcados en esta base de datos corresponde a anclas ubicadas en el sur de Inglaterra.

4. Para las referencias del gráfico se utilizaron como guía varios tratados (e.g. Cotsell, 1856; Réaumur y Duhamel de Monceau, [1764] 1993), diccionarios especializados (e.g. Blanckley, 1750, pp. 1,2; Chambaud y Des Carrières, 1815, ANCHOR; Dufief, 1810, p. 54) y estudios más recientes sobre la materia (e.g. Jobling, 1993 , pp. 136-138). Cabe notar que hay cierta ambigüedad con respecto a los nombres atribuidos a las partes de un ancla y en las equivalencias entre diferentes idiomas.
La pérdida de anclas debido a fallas o accidentes eran eventos recurrentes. Los esfuerzos mecánicos a los que estas piezas eran sometidas podían ocasionar la rotura de alguna de sus partes (ver más abajo). Por otro lado, los barcos que sufrían un temporal podían perder una o varias anclas, e incluso debían abandonarlas para darse a la vela. Situaciones de esta índole han quedado registradas en diarios de a bordo y otros relatos del período de interés (e.g. Bougainville, 1771; Dunmore, 1981; Duyker, 1992; por citar tres expediciones francesas de la segunda mitad del siglo XVIII) y están atestiguadas por cuantiosos ejemplares que descansan en el lecho de ríos y mares. Hoy en día, las anclas procedentes de sitios arqueológicos - unas aisladas, otras asociadas a restos de naufragios-constituyen una fuente de información de particular interés para el análisis de la tecnología ancorera y metalúrgica en general ${ }^{3}$.

\section{Las partes de un ancla de hierro y cepo de madera}

Las principales partes metálicas de un ancla eran las siguientes: 1) la caña o asta, que iba desde la cruz hasta el arganeo (o argolla), 2) los brazos, que nacían en la parte inferior de la caña (en la cruz) y ascendían en ángulo hacia los costados, 3) las palmas o mapas, a veces llamadas pestañas, orejas o manos, colocadas en la parte interior de los brazos y 4) el arganeo, argolla de donde pendía la pieza. En la Figura 1 están mencionadas las partes de un ancla del siglo XVIII ${ }^{4}$. 


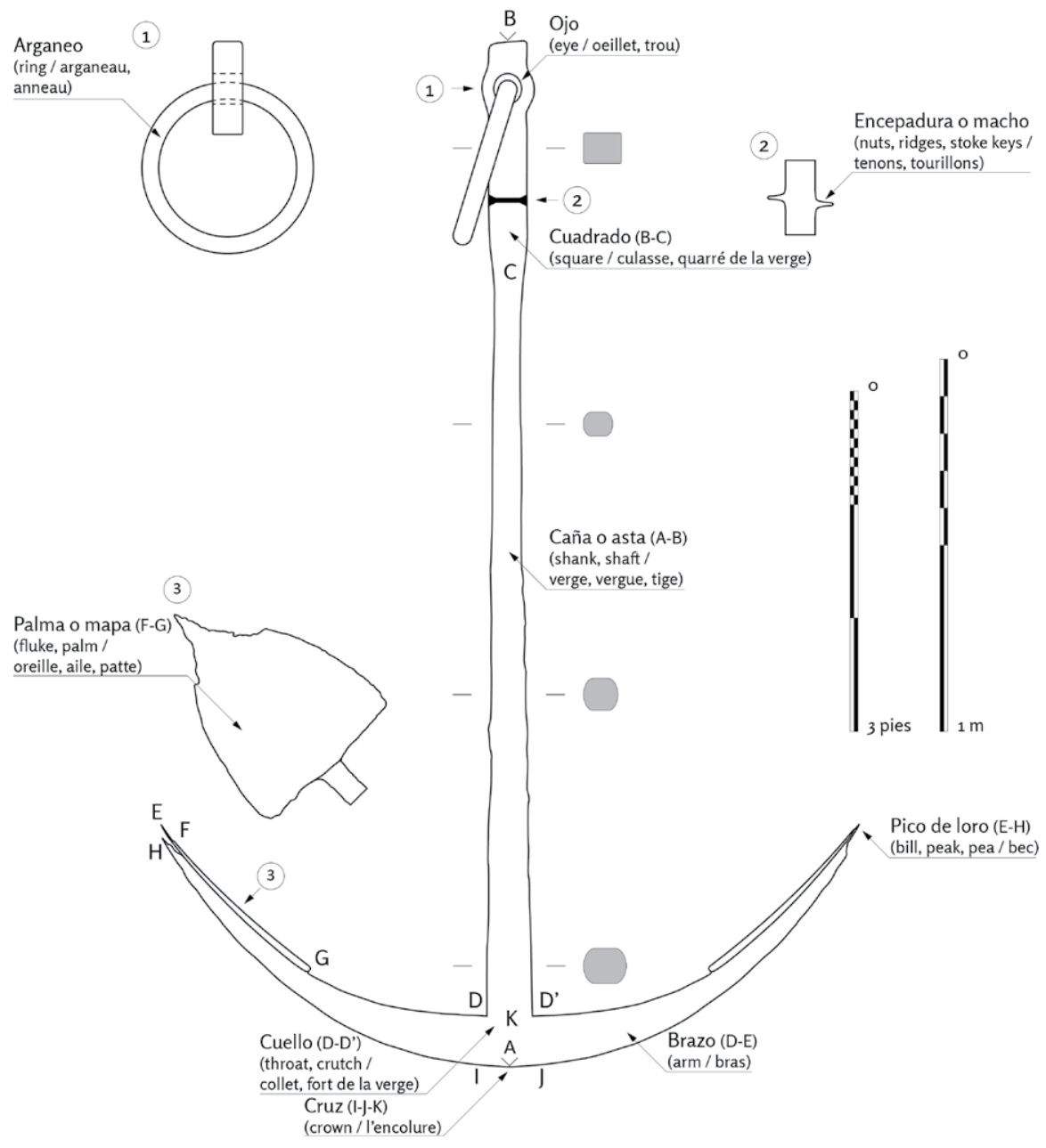

Figura 1. Nomenclatura de las principales partes de un ancla del siglo XVIII -el cepo de madera no está representado-que habría pertenecido al navío HMS Agamemnon (1781-1809). Nótese que el diseño difiere del de un ancla británica típica. La escala en pies corresponde a unidades inglesas (1 pie $=30,48 \mathrm{~cm}$ ) (Gráfico: N. Ciarlo, 2014, en base a una pieza expuesta en el Museo Naval de Montevideo, Uruguay).

La caña era la parte más alongada del ancla, en cuyo extremo inferior iban unidos los brazos y en el opuesto, el cepo. El largo, por lo general considerado desde la cruz hasta el extremo superior, guardaba cierta relación con la manga del barco. Durante la primera mitad del siglo XVIII, aún se utilizaba la antigua regla para calcular el largo del ancla de mayores dimensiones como $2 / 5$ de la manga del barco. Esta relación fue expresada en 1717 por William Sutherland en su obra Britain's Glory: or Ship-Building Unvaild (Sutherland, 1717, p. 22), aunque cambió luego por una menor. Al respecto, en su Traite du Navire, Bouguer sostuvo que el largo de la caña correspondía a $3 / 4$ de la manga (Bouguer, 1746, p. 98).

Los brazos eran las dos extremidades que permitían que el ancla quedara aferrada al fondo. Guardaban cierto ángulo con res pecto a la caña, del que dependía la operatividad del ancla: el enganche podía verse comprometido si el ángulo de los brazos era muy cerrado, mientras que el punto de unión con la caña podía ceder ante la tensión generada si el ángulo se abría en exceso. En la práctica solía buscarse una solución de compromiso. La apertura de los brazos, cabe anticipar, era ligeramente diferente según el lugar de fabricación y, además, fue cambiando a lo largo del tiempo.

Las palmas consistían en unas planchuelas de forma triangular que iban colocadas sobre la superficie interior de los brazos, desde cerca de la mitad hacia el extremo. Le 
5. A comienzos del siglo XIX, luego de varias experimentaciones, las cadenas de hierro comenzaron a utilizarse en los barcos de guerra británicos como complemento de los cables regulares. El reemplazo definitivo ocurrió años más tarde, en parte debido a la cautela del Almirantazgo frente al nuevo ingenio (Curryer, 1999, pp. 96-104).

6. El cepo de hierro fue introducido en la Real Armada británica hacia el último cuarto del siglo XVIII. Primeramente lo llevaron las anclas más pequeñas, i.e. los anclotes y algunas anclas de espía. Las anclas de mayores dimensiones continuaron utilizando el cepo de madera hasta entrado el siglo XIX. otorgaban mayor capacidad de agarre a las anclas una vez clavadas. El segmento distal de los brazos, inmediatamente a continuación de las palmas, se denominaba uña, y la punta propiamente dicha, pico de loro o de papagayo.

El arganeo era un aro de hierro, engastado a través del ojo en el extremo de la caña, al que iba amarrado el cable mediante malla de entalingadura. Esta argolla estaba recubierta por cabo, a fin de evitar que el hierro dañara al cable de cáñamo. Con la introducción de la cadena, fue necesario reemplazar el arganeo por un grillete ${ }^{5}$.

El cepo consistía en una pieza de madera compuesta que, orientada de forma perpendicular a la caña y al plano de los brazos, permitía que uno de estos últimos se aferrase al fondo y permaneciese en su lugar. Iba colocado en el extremo superior de la caña, que por debajo del ojo era escuadrada y tenía un resalto en forma de cuña (encepadura o macho) que contribuía a su encastre. Las mitades, usualmente de roble, se unían entre sí mediante cabillas y abrazaderas de hierro (Blanckley, 1750, p. 2; Falconer, 1780, ANCHOR; O'Scanlan, 1831, pp. 156, 238). Para prevenir su deterioro, el cepo solía forrarse con planchas de cobre o aleación de cobre, sistema similar al aplicado a los fondos de los barcos ${ }^{6}$.

Todas las partes de un ancla guardaban cierta proporcionalidad entre sí; por caso, en las anclas británicas el largo de la palma solía cubrir la mitad del brazo. No obstante, los criterios establecidos o sugeridos por distintos autores no eran idénticos. Además, en la práctica existían discrepancias entre piezas de diferentes astilleros o ancorerías coetáneos y, como se verá, las dimensiones y relaciones de tamaño entre las partes fueron sufriendo modificaciones a lo largo del tiempo (ver Telechea Idígoras, 1977, para el caso de las anclas españolas fabricadas en Hernani).

\section{Las anclas de un barco de guerra}

\section{Tipos, disposición y uso}

Un barco de guerra estaba equipado con varias anclas de distintos tamaños, conforme a su rango. En el caso de las fragatas y navíos, las cuatro que iban trincadas a proa, se denominaban de la siguiente manera (a partir de O'Scanlan, 1831, pp. 33-34):

" Ancla de leva, sencilla o de cabeza (small bower anchor, second anchor, ancre d’affourche): la más pequeña o de menor peso de las principales.

"Ancla de uso o del ayuste (best bower anchor, first anchor, seconde ancre): la que seguía en peso a la anterior. Ambas también eran llamadas de leva, de cabeza, de pendura o de servidumbre (bow anchors; ancres de bossoir, ancres de poste).

" Ancla de esperanza, horma, forma o formaleza (sheet anchor, ancre de miséricorde, ancre despérance, maitresse ancre, grande ancre): era la de mayor peso y se empleaba en caso de fuertes vientos o emergencia.

" Ancla cuarta o de respeto (fourth anchor o spare anchor; ancre de ramplacement, ancre de rechange): seguía en peso a la del ayuste, aunque podía igualar a la de esperanza, y por lo general se utilizaba como último recurso.

Las anclas de leva eran las empleadas corrientemente para fondear; se disponían en proa, suspendidas de unos pescantes (serviolas) que salían de las bordas del castillo, a los lados del bauprés. La del ayuste se llevaba en la banda de estribor y la sencilla, sobre la de babor. Las anclas de esperanza y de respeto iban trincadas por la parte exterior 
del costado, a popa de las de leva: la primera iba colocada a babor y la otra, a estribor? Los navíos de guerra solían llevar un ancla adicional denominada de la caridad o de la cala, más pesada que la de esperanza, que de ser necesario podía sustituir a cualquiera de las que estaban operativas (O'Scanlan, 1831, p. 34)

Además, los barcos tenían un ancla de espía (stream anchor; ancre à empeneller) y un anclote (kedge anchor; ancre de touée). Estas últimas eran más pequeñas que las anteriores y se utilizaban para anclar en aguas calmas, mover el barco en un puerto o río, sin suficiente espacio o viento, o mantenerlo alejado del cable de fondeo principal (Falconer, 1780,ANCHOR; Pering, 1819, p.15). En los botes y otras embarcaciones menores se utilizaban pequeñas anclas sin cepo y con cuatro o cinco brazos llamadas rezones (grapnel, grapling, grapin, hérisson, harpeaux). Un objeto parecido a estas últimas era el arpeo de rastrear (creeper, corbeau), que servía para recuperar objetos del lecho en aguas someras (ver Moore, 1801, GRAPNEL; O'Scanlan, 1831, pp. 52, 470; Steel, 1794, pp. 80, 82).

\section{Número de anclas llevadas a bordo}

En líneas generales, a mediados del siglo XVIII las fragatas y navíos de guerra británicos de tercer rango llevaban cuatro anclas principales, mientras que los de primer y segundo rango, iban equipados con cinco. Las corbetas, en cambio, contaban con tres anclas principales. Además, todos debían tener un ancla de espía y un anclote. En las dos décadas finiseculares, fragatas y navíos por igual transportaban cuatro anclas principales, con un peso acorde al rango de cada barco. Los navíos de primer y segundo orden pasaron a llevar un anclote adicional, más pequeño. Por caso, inicialmente el HMS Victory estuvo equipado con un ancla de esperanza, cuatro de leva, una de espía y dos anclotes (Jobling, 1993, pp. 98-100).

Sutherland consignó la cantidad y el peso de las anclas que debían llevar los barcos de guerra de la Real Armada británica de primer a sexto rango (Sutherland, 1766, p. 139). Los datos aportados por este autor dan cuenta de un estado previo a mediados del siglo XVIII, ya que se basó en las Reales Ordenanzas de 1744. La situación de los barcos de la flota hacia el último cuarto de la centuria está representada en la ordenanza de 1786 (ver Jobling, 1993). En la Tabla 1 se expone la información consignada posteriormente por Steel en The Elements and Practice of Rigging and Seamanship.

El peso de las piezas merece un comentario aparte. En 1750, Blanckley indicó que las anclas principales, i. e. la sencilla, del ayuste, de esperanza y de respeto, según las ordenanzas de 1744, debían tener el mismo peso. Por otro lado, la de espía y el anclote equivalían a un cuarto y un octavo del peso de aquellas, respectivamente (Blanckley, 1750, p. 2). Steel sostuvo luego que las anclas principales no diferían en su peso (Steel, 1794, p. 77). Una proposición semejante puede encontrarse en las obras de Moore y Pering (Moore, 1801: ANCHOR; Pering, 1819, p. 15). Al respecto, en un documento finisecular conservado en el National Maritime Museum (NMM), Greenwich, se ilustra un ancla de esperanza o de leva y un anclote, pertenecientes a un navío británico de 74 cañones (Figura 2). En el reverso de la lámina están detalladas las dimensiones de estas anclas junto con una tabla que contiene la cantidad, peso y costo de las piezas usadas habitualmente en un barco de aquel porte. La información consignada sugiere que no había diferencias entre las de esperanza y las de leva. En la práctica, sin embargo, solían existir discrepancias entre unas y otras (Jobling, 1993, p. 100).

A partir de los datos consignados en las ordenanzas de la Real Armada británica y de las características del equipamiento de diferentes barcos de guerra del siglo XVIII, que solía presentar discrepancias con relación a lo estipulado, puede apreciarse un incremento paulatino en el peso de las anclas. Acaso la expectativa era que, cuanto
7. Existen discrepancias en cuanto a la disposición que las anclas mantenían a bordo, conforme a la tradición de cada Armada (Steel, 1794, pp. 287, 288).

8. En el caso de los navíos franceses, el ancla de caridad iba colocada en posición vertical sobre la tablazón de la bodega o cala , delante de la poza de balas. Por caso, la pieza hallada en el navío francés Fougueux (1785-1805) podría corresponder a un ancla de este tipo (Rodríguez Mariscal, 2010, pp. 148-153). 


\begin{tabular}{|c|c|c|c|c|c|c|c|c|c|}
\hline \multirow{2}{*}{$\begin{array}{c}\text { Barcos }^{1} \\
\text { Cañones }\end{array}$} & \multicolumn{4}{|c|}{ Anclas } & \multirow{2}{*}{$\begin{array}{c}\text { Barcos } \\
\text { Cañones }\end{array}$} & \multicolumn{4}{|c|}{ Anclas } \\
\hline & \multirow{2}{*}{$\begin{array}{c}\text { No. } \\
5^{3}\end{array}$} & \multirow{2}{*}{\begin{tabular}{|l} 
Tipo \\
Leva
\end{tabular}} & \multicolumn{2}{|c|}{ Peso (cwt y qtr) } & & \multirow{4}{*}{$\begin{array}{c}\text { No. } \\
4 \\
1 \\
1\end{array}$} & \multirow{4}{*}{\begin{tabular}{|l} 
Tipo \\
Leva \\
Espía \\
Anclote
\end{tabular}} & \multicolumn{2}{|c|}{ Peso (cwt y qtr) } \\
\hline \multirow{3}{*}{110 y 100} & & & 81 & 0 & & & & 40 & 0 \\
\hline & 1 & Espía & 21 & 0 & 44 y 38 & & & 10 & 0 \\
\hline & 1 & Anclote & 10 & 2 & & & & 5 & 0 \\
\hline \multirow{3}{*}{98 y 90} & $5^{3}$ & Leva & 73 & o & & 4 & Leva & 39 & o \\
\hline & 1 & Espía & 18 & 0 & 36 & 1 & Espía & 9 & 0 \\
\hline & 1 & Anclote & 9 & $\mathrm{o}$ & & 1 & Anclote & 4 & 2 \\
\hline \multirow{3}{*}{80 y 74} & 4 & Leva & 71 & 0 & & 4 & Leva & 33 & 0 \\
\hline & 1 & Espía & 17 & 2 & 32 & 1 & Espía & 8 & 1 \\
\hline & 1 & Anclote & 8 & 2 & & 1 & Anclote & 4 & o \\
\hline \multirow{3}{*}{74} & 4 & Leva & 67 & 0 & & 4 & Leva & 31 & 0 \\
\hline & 1 & Espía & 16 & 0 & 28 & 1 & Espía & 8 & o \\
\hline & 1 & Anclote & 8 & o & & 1 & Anclote & 4 & 0 \\
\hline \multirow{3}{*}{64} & 4 & Leva & 57 & 0 & & 4 & Leva & 29 & 2 \\
\hline & 1 & Espía & 15 & o & 24 & 1 & Espía & 7 & 2 \\
\hline & 1 & Anclote & 7 & 2 & & 1 & Anclote & 3 & 2 \\
\hline \multirow{3}{*}{60} & 4 & Leva & 53 & 0 & & 4 & Leva & 25 & 0 \\
\hline & 1 & Espía & 12 & 0 & 20 & 1 & Espía & 7 & 2 \\
\hline & 1 & Anclote & 6 & 0 & & 1 & Anclote & 3 & 2 \\
\hline \multirow{3}{*}{50} & 4 & Leva & 49 & 0 & & 3 & Leva & 20 & 0 \\
\hline & 1 & Espía & 11 & 0 & 14 & 1 & Espía & 7 & 0 \\
\hline & 1 & Anclote & 5 & 2 & & 1 & Anclote & 3 & 2 \\
\hline
\end{tabular}

Tabla 1. Cantidad y peso de las anclas de los barcos de guerra británicos (Steel 1794, p. 81). Referencias: 1) no fueron reproducidas las cifras correspondientes a las corbetas y bergantines de $200 \mathrm{t}, 2) \mathrm{cwt}$ : hundred weight o centum weight (quintal), equivalente a $112 \mathrm{libras} 0 \mathrm{ca}$. $50,8 \mathrm{~kg}$, qtr: quarter (arroba), equivalente a 28 libras o ca.12,7 kg, 3) la reducción del número de anclas de leva en navíos de primer y segundo rango (de 5 a 4 anclas) figura en las ordenanzas de 1786 (Jobling, 1993, p. 103).

9. El diseño no es indicativo del contexto de uso de una pieza.

Por ello, en un sitio arqueológico, tampoco puede considerarse a priori como evidencia de la procedencia de restos materiales asociados. más robustos, estos elementos podrían soportar mayores esfuerzos. Este hecho, a su vez, estuvo relacionado con el aumento del tonelaje y poder de fuego que fueron experimentando las naves a lo largo del tiempo (Jobling, 1993, p. 111).

\section{Los modelos europeos: rasgos diagnósticos}

En la introducción de A Treatise on the Anchor, Pering resaltó que ninguna nación adoptó un ancla similar en cuanto a su forma, proporciones y partes componentes (Pering, 1819, p. 9). Esta situación, aunque lamentable a los ojos del autor, es la razón por la que hoy en día es posible identificar la procedencia de un ancla, i. e. al lugar de fabricación, a partir de sus rasgos morfológicos ${ }^{9}$. Ello dependerá, por supuesto, de la regularidad o grado de estandarización de las piezas. Por otro lado, las modificaciones en el diseño de las anclas pueden resultar de utilidad como indicadores cronológicos aproximados. Ambos aspectos resultan de sumo interés en el marco de una investigación arqueológica, en particular en sitios de naufragios, dada la elevada representatividad con que cuentan las anclas.

\section{Morfología y dimensiones}

En la época que nos ocupa, dado su grado de estandarización, destacan las anclas de la Real Armada británica. El patrón conocido como Old Admiralty Longshank fue 
A
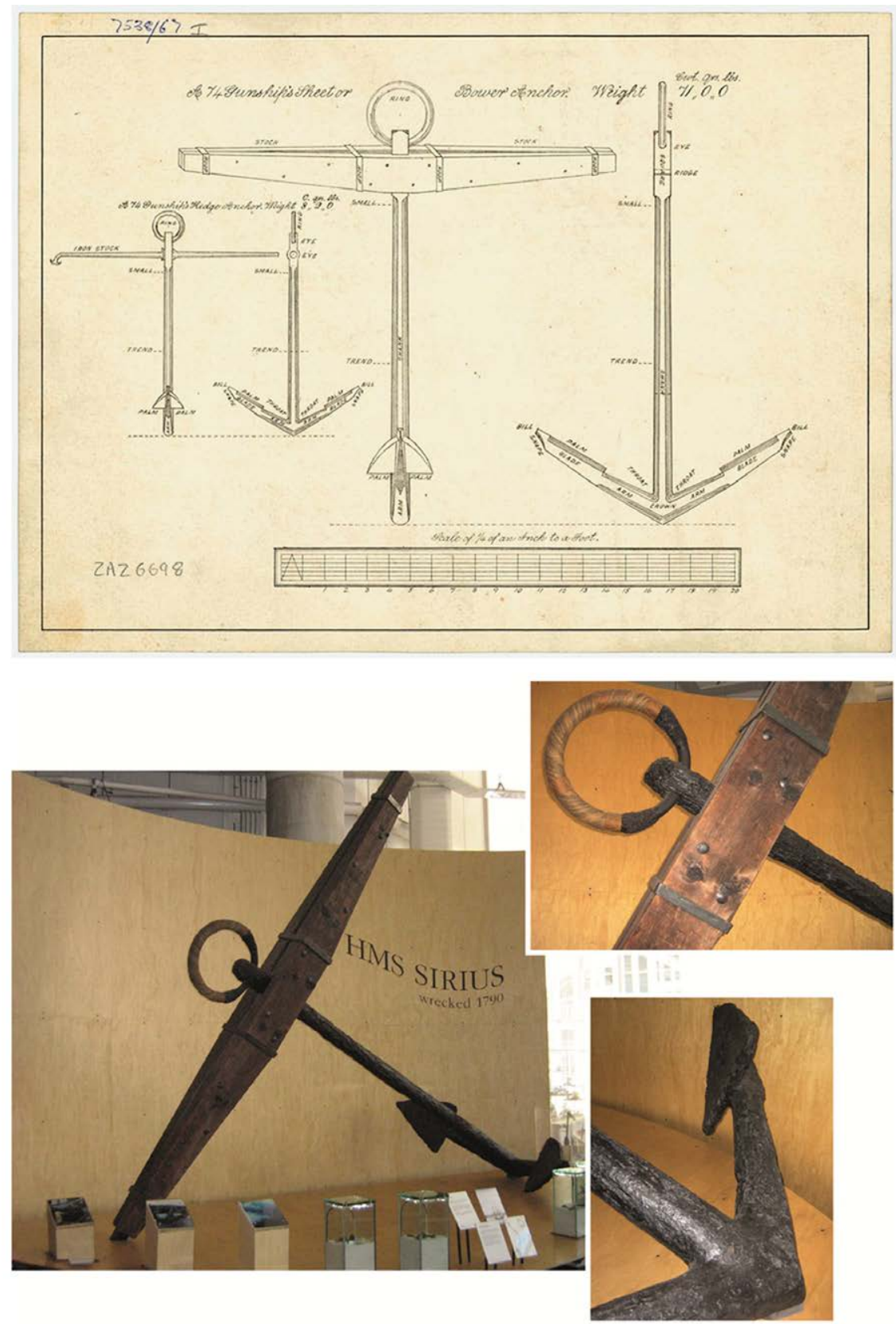

Figura 2. A) Vista de planta y perfil de un ancla de leva de 71 quintales y un anclote de ocho quintales pertenecientes a un navío de 74 cañones, firmado por C. S. Thomas, ca. 1790. Documento: NMM, ZAZ 6698. B) Ancla de leva del sitio HMS Sirius (1780-1790), localizada en la década de 1980 y exhibida en el Australian National Maritime Museum, Sydney. Nótense los rasgos típicos de las anclas británicas de la época (ver más abajo). El cepo es una reproducción (Fotos: C. Perfumo, 2010).

utilizado, con mínimas variaciones, para equipar los barcos de guerra británicos desde mediados del siglo XVI hasta principios del siglo XIX, cuando fue reemplazado por el modelo que patentó Richard Pering en 1813 (Curryer, 1999, pp. 73-77). Uno de los planos más tempranos de este tipo de anclas fue presentado por Sutherland, en la obra ya mencionada. Los rasgos diagnósticos reconocibles a simple vista eran: la cruz con terminación puntiaguda, los brazos rectos y el ángulo cerrado que formaban con la caña, el modo de unión de los brazos, los mapas triangulares (de morfología equilátera) y la sección cuadrada de la caña debajo del ojo (ver Figura 2). 


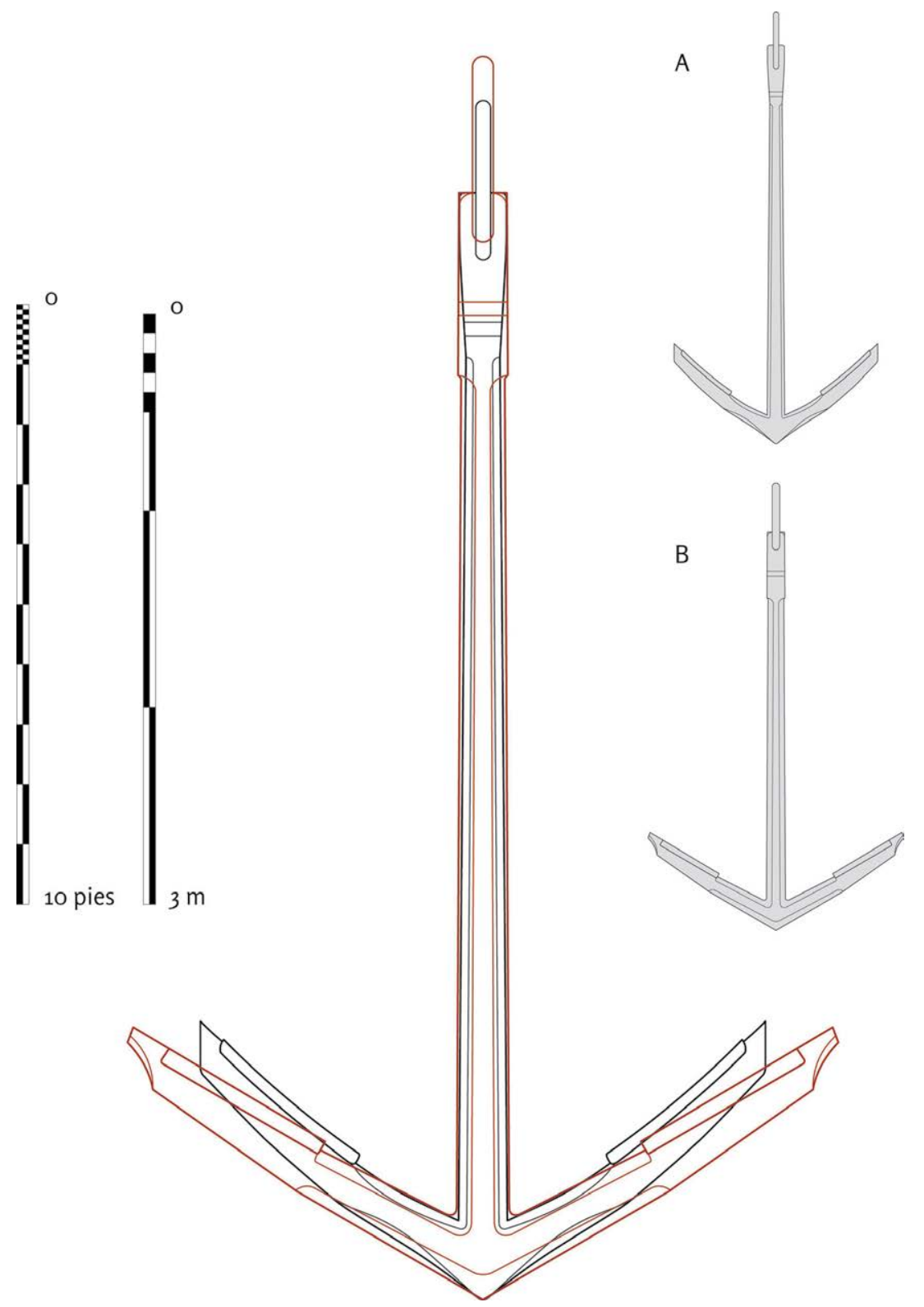

Figura 3. Comparativa entre las siluetas de dos anclas británicas del siglo XVIII, ilustradas en: A) Sutherland (1717, p. 23), B) plano del NMM, ZAZ 6698, mencionado más arriba. Aunque el tamaño y peso de las dos piezas son similares, el ángulo de los brazos es más obtuso en el ancla de ca. 1790. La escala de la izquierda está expresada en pies ingleses (Gráfico: N. Ciarlo, 2014).

En este tipo de anclas, el largo de la caña solía guardar relación con la manga del barco. En su tiempo, Pering realizó un recuento de las proporciones que diferentes autores habían propuesto. Por lo general, el valor se encontraba entre $1 / 2$ y 3/8 de la manga. Asimismo, este autor notó que los marineros tenían distintos modos de precisar el peso, por ejemplo aplicando una relación a partir del largo de la caña (Pering, 1819, p. 19). Las partes de un ancla también guardaban cierta proporcionalidad entre sí: el largo de los brazos equivalía a un tercio del de la caña (Pering, 1819, p. 16), mientras que las palmas medían aproximadamente la mitad de los brazos (Steel, 1794, p. 79). El ángulo de estos últimos también era un rasgo significativo. Sutherland propuso un valor que - según se desprende de su explicación, no así del gráfico - debía calcularse así: partiendo del largo del brazo (ver Figura 1, distancia D-E) se formaba un triángulo equilátero, tomando a aquel como base de la figura, que se rotaba sobre el punto de 


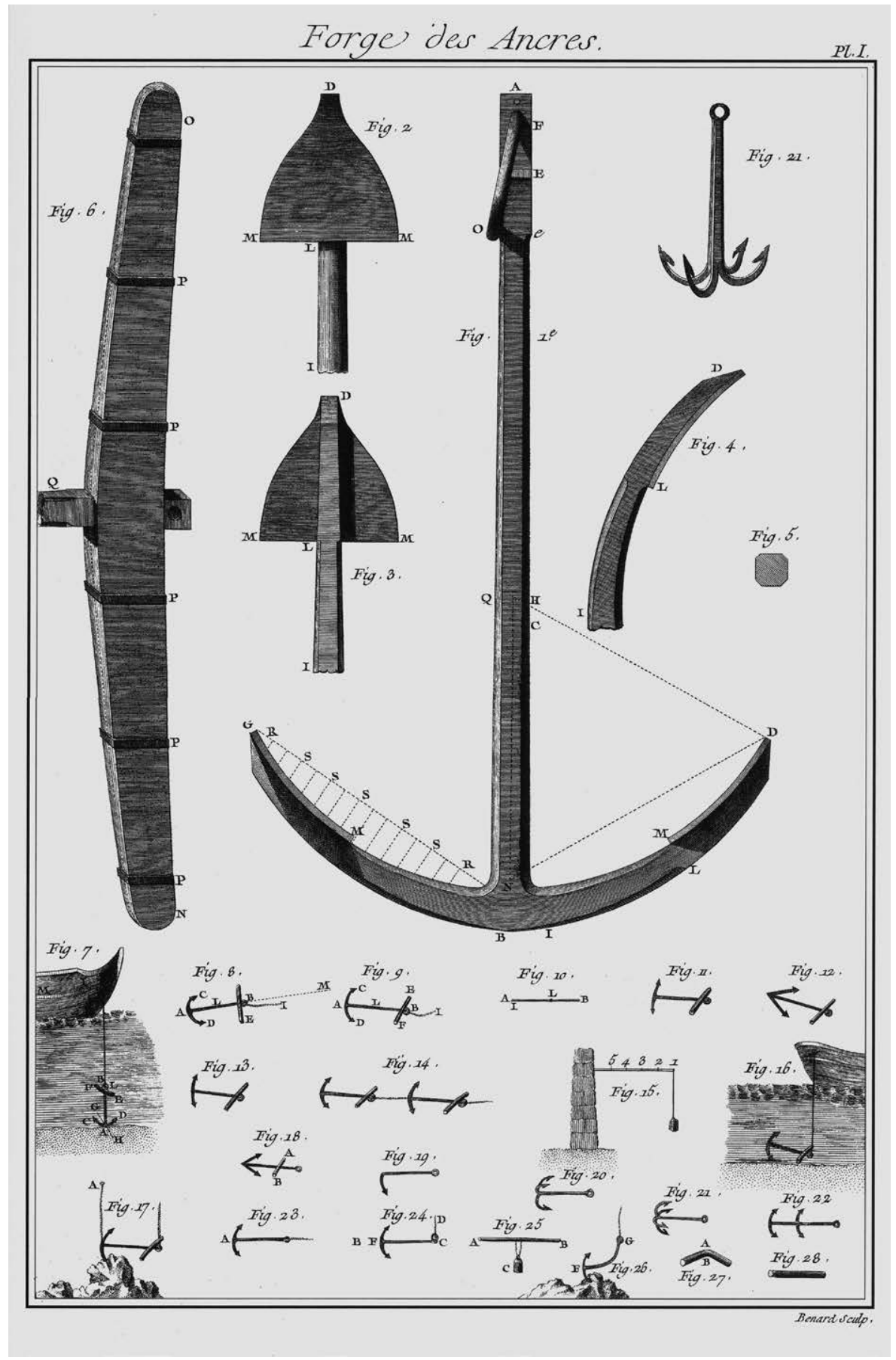

Figura 4. Lámina primera de la obra Fabrique des Ancres, donde se muestran las partes de un ancla francesa, ca. 1760 (Réaumur y Duhamel de Monceau, [1764] 1993, lámina 1).

unión entre el brazo y la caña (ver Figura 1, nodo D) de modo tal que el vértice opuesto a la base del triángulo coincidiera con el eje longitudinal de la caña. Sutherland llamó middle meeting a este punto medio. Las opiniones en torno al modo de posicionar los brazos, comentó, fueron siempre dispares: otros utilizaron como punto de intersección con la caña su lado interno o externo. Por tanto, siguiendo uno u otro criterio, se obtenía un ángulo más o menos agudo, respectivamente (Sutherland, 1717, pp. 22, 23). Hacia la segunda mitad del siglo XVIII, los brazos de las anclas británicas estaban posicionados a $60^{\circ}$ (Steel, 1794, p. 79). Por ejemplo, los brazos del ancla de esperanza 
10. Los gráficos corresponden a piezas de tamaño similar: la primera pesa 71 quintales y dos arrobas y la caña mide 18 pies y seis pulgadas, mientras que la segunda pesa 71 quintales y el largo es 18 pies y cinco pulgadas. A principios del siglo XVIII, cabe notar, la primera servía para equipar un barco de mayor porte.

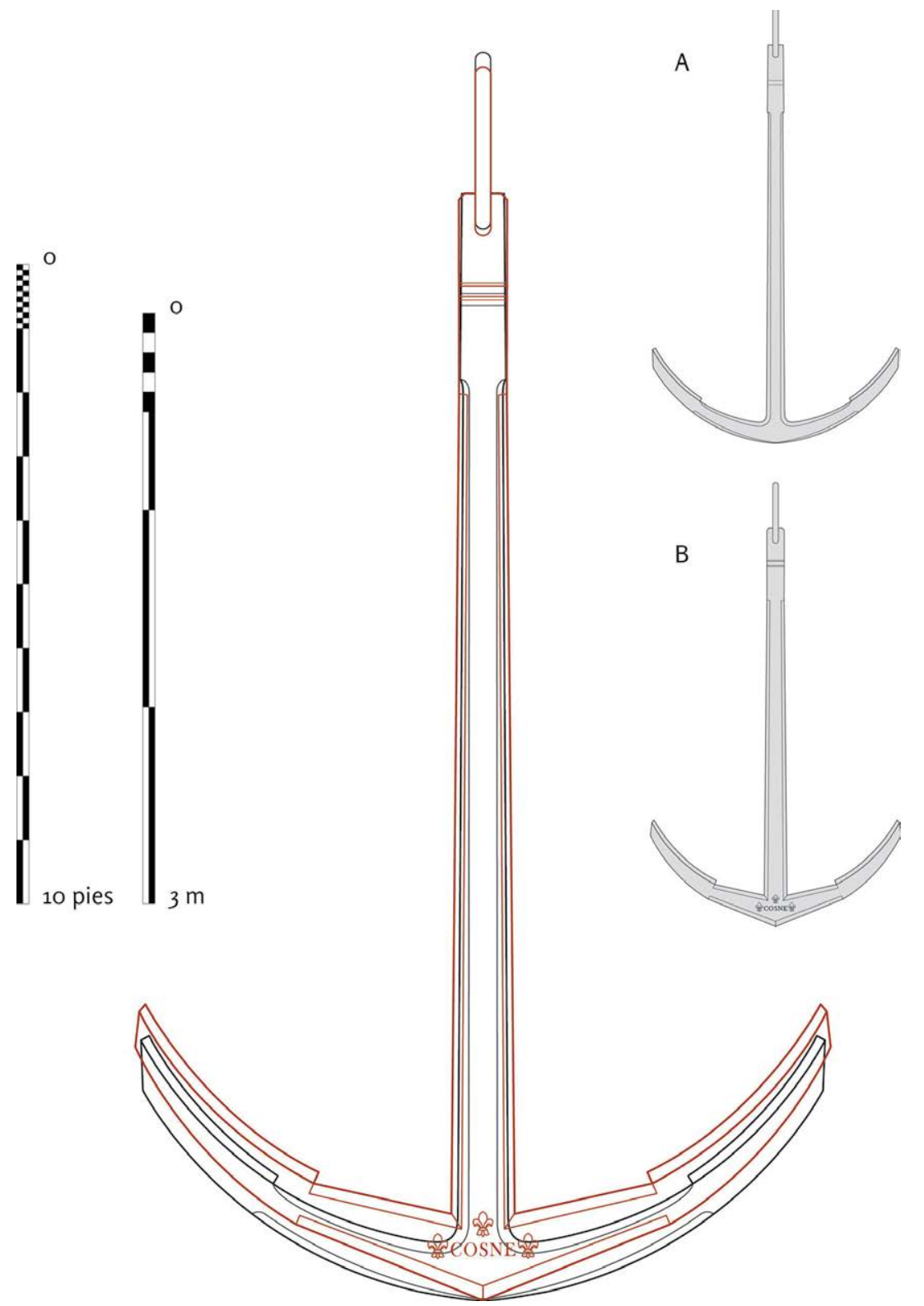

Figura 5. Comparativa entre dos anclas francesas del siglo XVIII. En esta lámina se aprecian intercalados los perfiles de dos anclas francesas, a partir de: A) Réaumur y Duhamel de Monceau ([1764] 1993, lámina 1), B) Diderot y d'Alembert (1769, Marine, Forge des Ancre, lámina 12). La pieza $B$ es ligeramente más robusta y, pese a que sus brazos nacen rectos, luego adoptan una forma curvada. La escala de la izquierda está expresada en pies franceses (Gráfico: N. Ciarlo, 2014).

original del HMS Victory tenían un ángulo de $c a .59^{\circ}$, que se cerraba unos $7^{\circ}$ a la altura de la palma (Jobling, 1993, p. 93). En líneas generales, el ángulo propuesto por Sutherland permaneció vigente durante décadas, si bien una comparación de las representaciones históricas sugiere que experimentó un leve aumento a lo largo del siglo XVIII (Figura 3) ${ }^{10}$.

El ancla ilustrada en la obra Fabrique des Ancres, que presentó Réaumur — con notas y adiciones de Duhamel- en 1723 ante la Académie Royale des Sciences de París (Réaumur y Duhamel du Monceau, [1764] 1993, lámina 1), permite distinguir qué diferencias existían entre las anclas británicas y francesas. Las anclas galas del siglo XVIII tenían los brazos curvos y las palmas se extendían hasta su extremo copiando 
la curvatura (Figura 4). En la referida lámina, los brazos esbozan un ángulo de $60^{\circ}$ y el triángulo que se forma en este caso es equilátero. En cuanto al cepo, los maderos tenían forma curva y sus extremos redondeados ${ }^{11}$. Bouguer también describió la morfología y proporciones de este tipo de anclas. Con respecto a los brazos, señaló que formaban entre sí un arco de $120^{\circ}$ (Bouguer, 1746, p. 97). Aunque, según este autor, las palmas tenían un largo similar al de las anclas británicas, en el ancla ilustrada en la sección Marines, Forge des Ancres de La Enciclopedia de Diderot y d'Alembert, presentan una extensión superior. Al respecto, cabe notar, una palma amplia le confería al ancla una mejor capacidad para aferrarse al fondo, pero a la vez implicaba que debía realizarse un mayor esfuerzo para arrancarla.

El ángulo de los brazos, que en teoría era semejante al de las anclas británicas, podía presentar ligeras variaciones. Por caso, una de las anclas de leva del navío francés de 74 cañones Fougueux (1785-1805), que yace in situ, exhibe una apertura de brazos en torno a los 55 (Rodríguez Mariscal, 2010, p. 150). Entre otros casos arqueológicos de origen francés, pueden citarse las piezas del Queen Anne's Revenge (ca. 1713-1718), ex-Concorde (Lusardi, 2000, p. 61), y de la fragata La Surveillante (1778-1797) (Breen y Forsythe, 2007, pp. 44, 49). Pese a que existían ligeras discrepancias entre las anclas francesas de la época, grosso modo respetaban un diseño semejante (Figura 5).

En este punto es preciso aclarar que la información consignada en las fuentes documentales, a la fecha de su publicación, podía ser algo anticuada. El caso más notable es el de la ya referida sección de La Enciclopedia, basada en la memoria sobre anclas de François Trésaguet, que fue contratista de la Armada (ver Pinault-Sørensen, 1992).

Con relación a las anclas de origen español, las anclas fabricadas en Hernani hacia mediados del siglo XVIII reúnen las características típicas de las piezas de este país. Aunque existían ligeras variaciones, según los reportes de aquel entonces, en líneas generales la caña solía triplicar el largo del brazo, mientras las palmas sobrepasaban ligeramente la mitad de este último ${ }^{12}$. El diseño de las anclas españolas se mantuvo sin mayores cambios durante el período de interés. Cabe señalar que, hacia la segunda mitad de la centuria, hubo propuestas para mejorar la morfología y prestaciones de los elementos de fondeo. Muestra de ello es una carta dirigida por Jorge Juan a Julián de Arriaga el 18 de abril de 1765, en la que expone su parecer acerca de las supuestas ventajas que revisten las anclas fabricadas por un maestro herrero de Cartagena frente a las utilizadas habitualmente ${ }^{13}$. En el documento anexo se aprecia la morfología de las nuevas anclas y anclotes propuestos para los barcos de la Real Armada (Archivo General de Simancas, Secretaría de Marina, No. 00606). Las piezas ilustradas en este último únicamente difieren en su tamaño y guardan notables semejanzas con el ancla de La Enciclopedia, sobre todo en la forma de la corona y curvatura de los brazos. La principal diferencia yace en las palmas, que en las anclas españolas son más cortas y, al igual que en el caso de las británicas, tienen uñas (segmento puntiagudo en el extremo de los brazos). La sección de la caña era otro rasgo que se pretendía innovar: su forma cuadrada o cuadrilonga permitiría extender la longitud de las anclas sin alterar el peso y, de este modo, mejorar su capacidad para tenderse sobre el fondo. Si bien Jorge Juan estaba de acuerdo con el cartaginés en este respecto, también notó que supondría una menor fortaleza con respecto a las anclas utilizadas corrientemente en España, que tenían caña de sección circular u oval. Debido a ello y a la voz de la experiencia, que no suministraba razones para cambiar el diseño probado con éxito durante tanto tiempo, sostuvo el ilustre marino que las anclas habrían de seguir construyéndose como era habitual.

Volviendo a la cuestión de la identificación de la procedencia de las anclas y teniendo en cuenta lo expuesto hasta ahora, puede reconocerse a simple vista un notable contraste a nivel morfológico entre un ancla británica y una francesa (Figura 6).
11. Los cepos de las anclas inglesas se mandaron hacer con extremos redondeados a partir de 1780 , debido a los daños que estos maderos ocasionaban sobre el forro de cobre de los cascos (Jobling, 1993, pp. 103-104).

12. En el libro de Telechea Idígoras (1977) se citan numerosos documentos que dan cuenta de ello.

13. La transcripción del documento original fue gentilmente suministrada por Sergio López Martín, que hizo uso de esta en su trabajo de fin de máster (López Martín, 2017) 


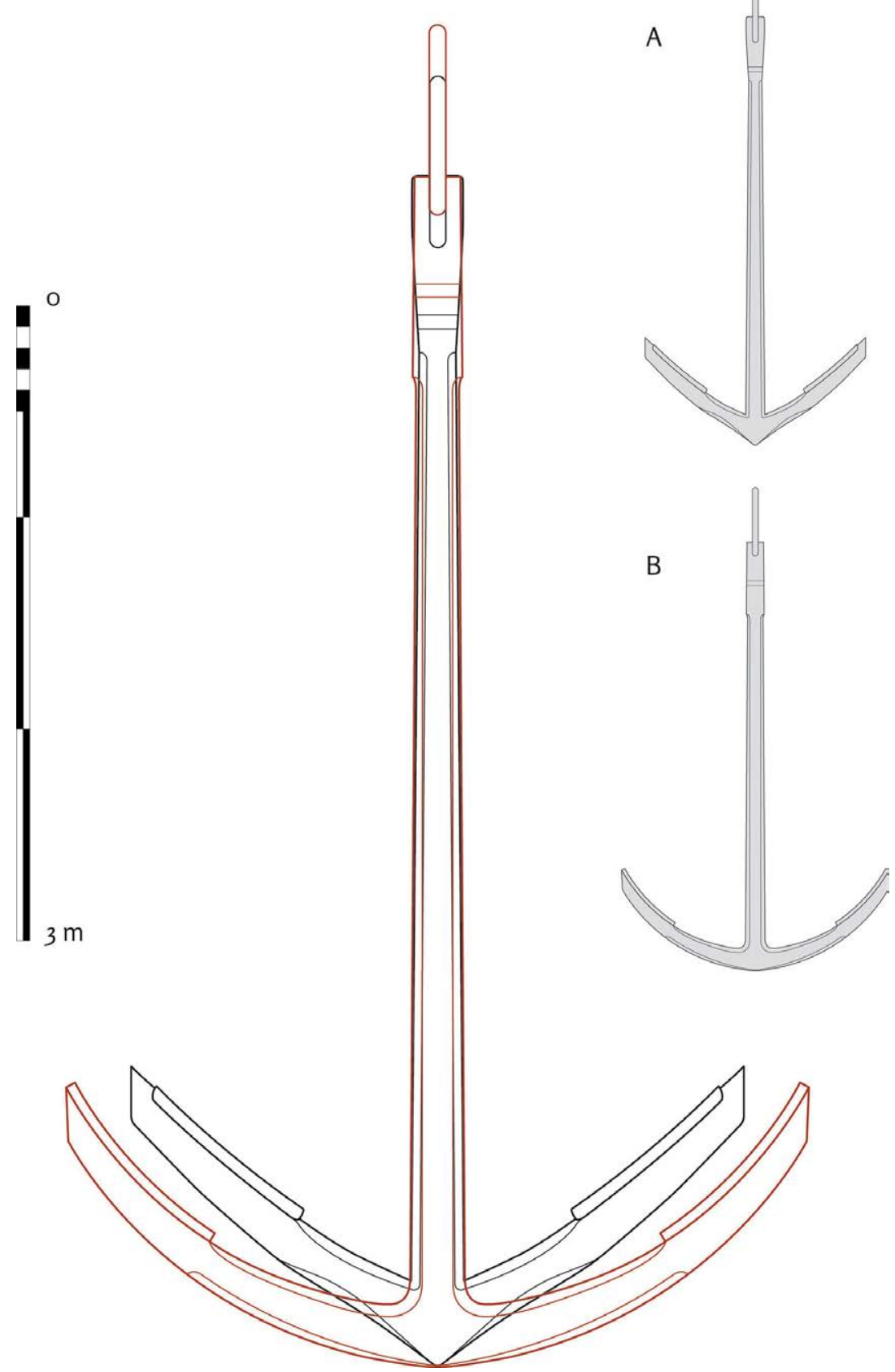

Figura 6. Comparativa entre anclas inglesas y francesas del primer tercio del siglo XVIII, reproducidas a partir de: A) Sutherland (1717, p. 23), B) Réaumur y Duhamel de Monceau ([1764] 1993, lámina 1). La principal diferencia radicaba en la forma de los brazos y el ángulo de estos con respecto a la caña (Gráfico: N. Ciarlo, 2014).

$\mathrm{Al}$ analizar este tipo de piezas es preciso tomar ciertos recaudos. Como fue anticipado, más allá de los aspectos típicos de cada modelo, podían existir discrepancias asociadas a la fabricación de cada lugar. Las anclas Old Admiralty Longshank del sitio HMS Sirius (1790) presentan varias diferencias en referencia al largo de la caña, la forma y extensión de los brazos y el grueso de la corona (Stanbury, 1994, p. 71). Además, muchas anclas sirvieron para equipar distintos barcos, no siempre del mismo pabellón. Las situaciones de transferencia de estos objetos eran varias, e. g. compra, botín y salvamento. Si se cuenta con evidencia adicional sobre la nacionalidad de un naufragio, es posible evaluar aspectos de esta índole, no así en el caso de los hallazgos aislados. 


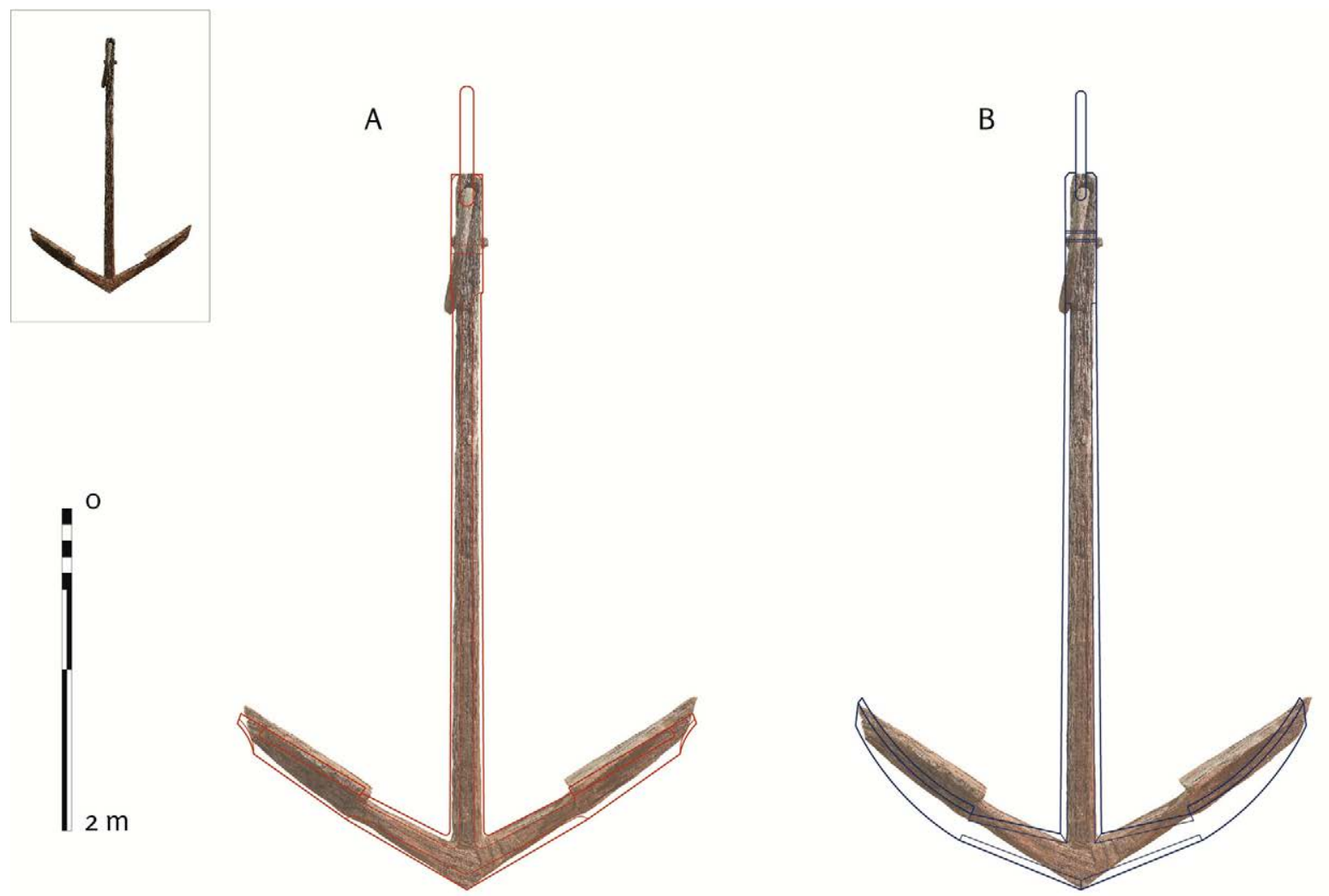

Figura 7. Imagen de una de las anclas halladas en la Bahía Doubtless, atribuida al St. Jean Baptiste. A la imagen de la pieza se superpone el diseño de dos anclas: A) británica, en base al documento ZAZ 6698 antes referido, ca. 1790, B) francesa, en base a Diderot y d'Alembert (1769, Marine, Forge des Ancres, lámina 12) (Gráfico: N. Ciarlo, 2015).

Las anclas del barco francés St. Jean Baptiste ilustran esta última situación. En 1769, el explorador Jean de Surville partió en esta nave desde Puducherry hacia el Pacífico Sur. Para su desgracia, la expedición fue en fracaso. Gran parte de la tripulación murió y apenas lograron llegar a la costa occidental de América, donde el barco fue apresado por los españoles y debió esperar tres años antes de regresar a Francia. A finales de la década de 1960, en la Bahía Doubtless, Nueva Zelanda, sitio adonde la tripulación había sufrido una fuerte tormenta, se localizaron dos anclas y un rezón que fueron atribuidos al St. Jean Baptiste (Tarlton, 1977). Una de las dos anclas de mayor portese encuentra exhibida en el Museum Te Papa Tongarewa, Wellington. Está catalogada bajo el No. NS000047/2 como ancla de hierro forjado, origen francés, $c a$. 1760, ancorero desconocido, largo de la caña: 444,5 cm, donada en 1974 por Mike Bearsley y Kelly Tarlton. El diseño del ancla en cuestión, sin embargo, responde a un modelo británico de la segunda mitad del siglo XVIII (Figura 7).

\section{Marcas superficiales}

Además de las características morfológicas, entre las peculiaridades diagnósticas pueden contarse las marcas de fabricación. Estas se plasmaban en determinados sectores del ancla y la cruz era uno de los sitios por excelencia. Destacan en este sentido las anclas francesas, que solían llevar en una de las caras el nombre del fabricante y distintivos nacionales como la flor de lis (fleur-de-lys) y en la otra, el peso expresado en libras (Rodríguez Mariscal, 2010, p. 151).

Las anclas británicas destinadas al servicio naval debían estar marcadas con la flecha del Almirantazgo y las iniciales del ancorero contratado. No obstante, ante las reiteradas 


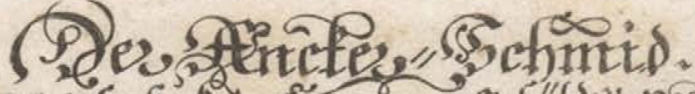

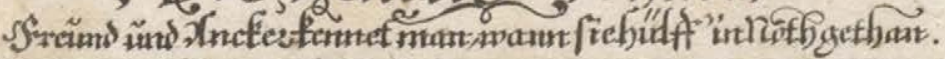

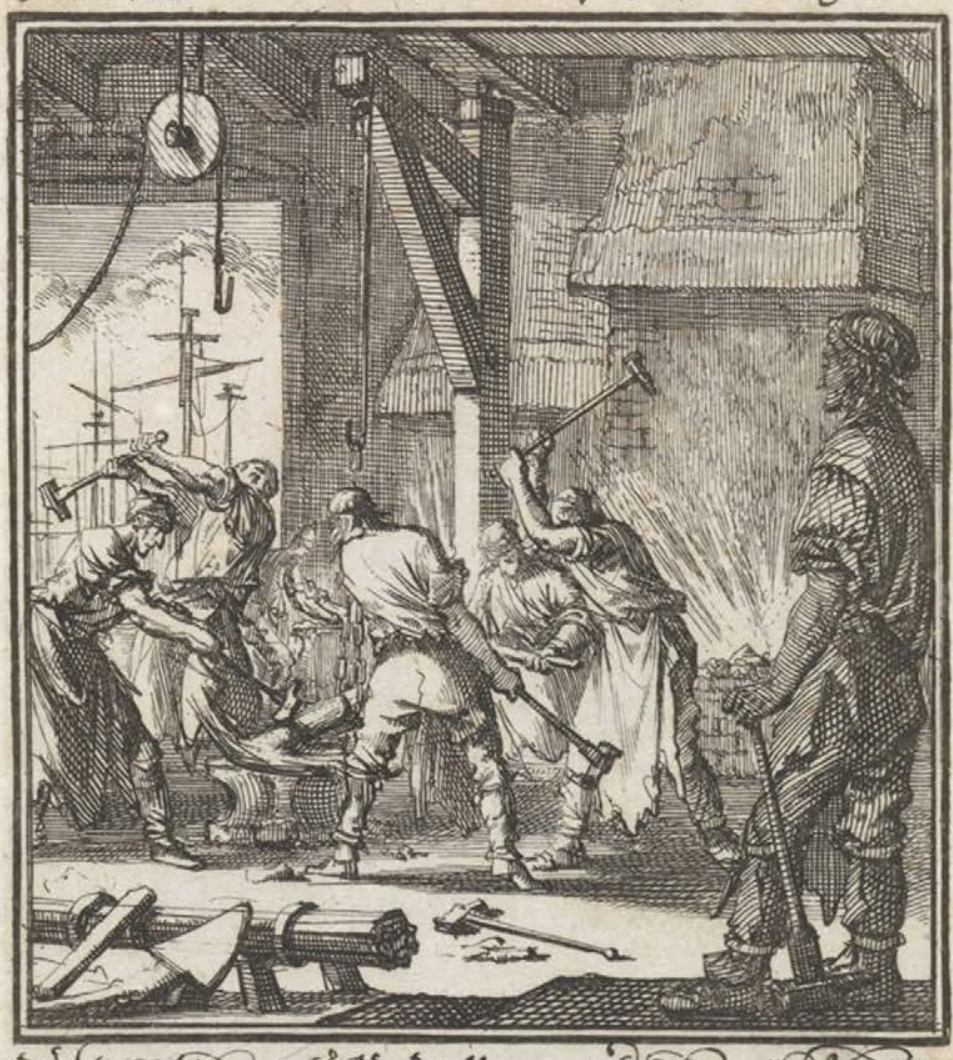

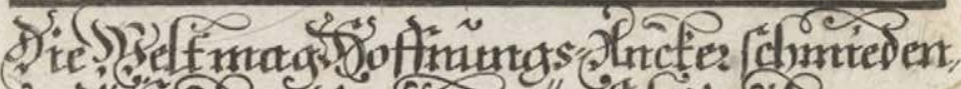

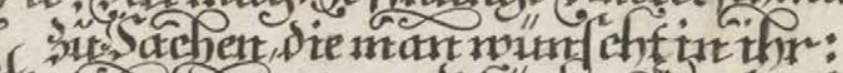

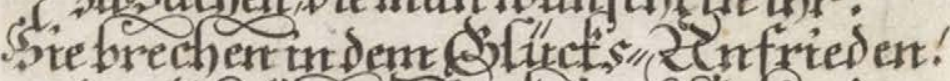

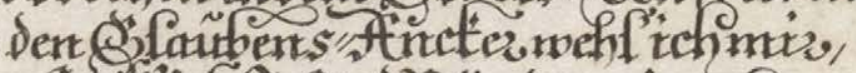
denfenctichgefits situder eir, fouffrintosostumel mein.

A

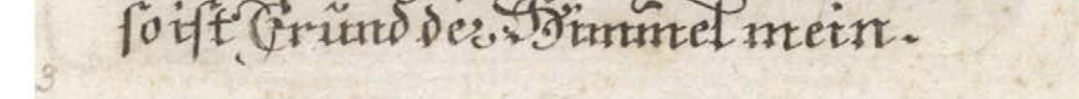

B

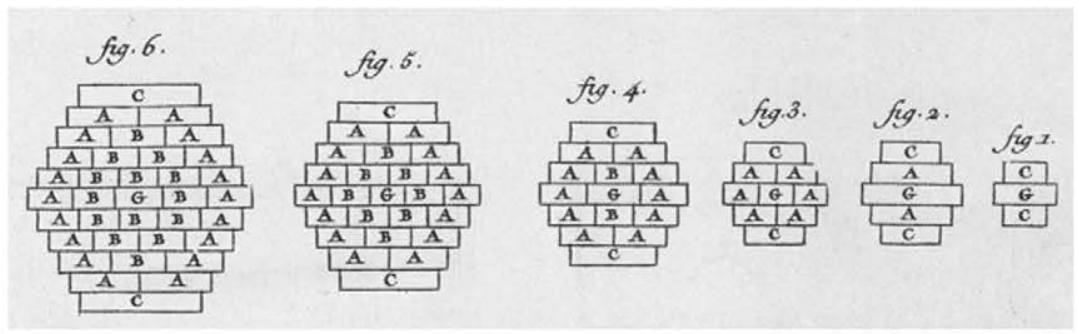

Figura 8. A) Ankersmid (1698): interior de una ancorería holandesa. Grabado anónimo, impreso por Jan Luyken, Ámsterdam. Documento: RP-P-1896-A-19368-160o, Rijksmuseum (Museo Nacional de Ámsterdam), Países Bajos. B) Configuración de los paquetes de barras de hierro (corte transversal) utilizados para fabricar anclas de: 1) $100 \mathrm{a} 200 \mathrm{lb}$; 2) $300 \mathrm{a} 400 \mathrm{lb}$; 3) 500 a $800 \mathrm{lb}$; 4) $900 \mathrm{a} 2.000 \mathrm{lb}$; 5$) 2.100 \mathrm{a} 5.000 \mathrm{lb}$ y 6$) 5.100 \mathrm{a}$ $8.000 \mathrm{lb}$. Las letras indican el tipo de barra, a saber: (a) barres à talon, (b) barres du milieu, (c) couvertures $y(g)$ gouvernail (Diderot y d'Alembert, 1769, Marine, Forge des Ancres, lámina 6). 
fallas que experimentaban, en parte debido a las limitaciones de la tecnología de forjado disponible, los fabricantes eran reacios a plasmar sus nombres (Jobling, 1993, p. 124). Casos que ilustran el empleo de la referida flecha son el ancla del HMB Endeavour (1764-1778), barco comandado por James Cook en su primer viaje al Pacífico, y el ancla que perdió el navío de 64 cañones HMS Dictator hacia 1814 en Point Patience, Maryland, EE.UU. También pueden encontrarse grabadas marcas tales como el peso, el lugar de producción y el barco al que pertenecían. La caña del ancla de aquel navío llevaba la inscripción Rec Chat $6 \times 3 \times 24$, léase que la pieza fue recibida en Chatham - puede afirmarse que fue fabricada por contrato-y pesaba $6 \mathrm{cwt}$, 3 qtr y $24 \mathrm{lb}$ (ca. 354 $\mathrm{kg}$ ). El cepo de madera, que se encontró en muy buen estado, tenía las siguientes marcas: Dictator (nombre del barco) $6 \times 3 \times 24$ (peso) y 6625 (número de pieza) (Knuckey, 1988; Schwartz y Green, 1962).

Debido a los procesos de deterioro que sufre el hierro forjado en el medio acuoso, especialmente salobre, habitualmente estos rasgos no son reconocibles a simple vista en las piezas procedentes de sitios arqueológicos.

\section{Tecnología de producción}

\section{Las fábricas y los maestros ancoreros}

La producción de anclas estaba a cargo de herreros especializados, los maestros ancoreros, e incluso de los ship smiths, que eran los responsables de hacer los herrajes y herramientas necesarios para la construcción de barcos (Brack, 2008, p. 13). En general, se fabricaban en establecimientos especializados, llamados ferrerías o ancorerías. Estos sitios tenían diferentes características, según el equipamiento que empleaban (e. $g$. martinetes hidráulicos, pescantes, fraguas, etc.) y el porte de las anclas que podían producir. En la Figura 8 se enseña el interior de un taller holandés de finales del siglo XVII, donde hay un grupo de operarios forjando un ancla pequeña a fuerza de brazo, junto a la fragua y al pescante empleado para mover las piezas. En primer plano es posible reconocer a otro individuo, posiblemente el maestro ancorero, y un haz de barras de hierro, con las que se confeccionaban las partes principales de las anclas (ver más abajo).

En La Enciclopedia se muestra cómo era el interior de una ancorería alrededor de mediados del siglo XVIII. A diferencia del caso anterior, tanto el mazo (martinete) como los fuelles estaban accionados por medio de energía hidráulica. Este equipamiento requería, por un lado, que la fábrica se encontrara próxima a un curso de agua permanente y, por el otro, de una considerable inversión inicial de capital. La producción tendía a estar concentrada en un número reducido de establecimientos de estas características, que empleaban gran cantidad de hombres. Estas ancorerías funcionaron principalmente en Inglaterra y Francia. En España, en cambio, las anclas para los navíos se fabricaron en talleres más modestos, en lo que a cantidad de personal e instrumental respecta, y sobre la base de conocimientos y prácticas de fuerte raigambre local.

En Inglaterra, la producción de anclas para los barcos de guerra estuvo, con algunas excepciones, bajo control directo de la Armada. A principios del siglo XVIII, el Consejo Naval resolvió que los ancoreros contratados trabajasen de forma permanente en los astilleros, supervisados por oficiales del gobierno. Además, se sugirió que la producción de las piezas de mayor tamaño estuviera exclusivamente a cargo de la Armada y que las ancorerías privadas se ocuparan de suministrar las restantes. Durante la primera mitad de la centuria, el astillero de Deptford lideró la producción. En 1748, allí había tres maestros ancoreros, 12 contramaestres y 88 maceros o martilladores. En las décadas siguientes, ante la creciente demanda de anclas para los nuevos barcos, se expandió 
notablemente la producción en otros astilleros estatales, i.e. Chatham, Woolwich, Portsmouth, Plymouth y Sheerness (Jobling, 1993, pp. 86, 87, 95).

En el caso de Francia, las principales forjas donde se producían anclas para abastecer a la Marina de Guerra durante el siglo XVIII fueron las de Cosne y Guérigny, en Nièvre, Borgoña. En esta última localidad, rica en minerales de hierro y bosques, el maestro de forja Babaud de la Chaussade (1706-1792) montó las Forges Royales, que fueron adquiridas por el rey antes de la Revolución, en 1781 (Taillemite, 2003, p. 455). En Cosne, por otro lado, trabajó el renombrado ancorero François Trésaguet, al que se hizo referencia más arriba (ver Gay, 1997).

España también contó con varias instalaciones. En 1751, en el marco del plan de renovación naval que emprendió el Marqués de la Ensenada, Juan Fernández de Isla montó en Marrón una usina adonde se forjaron anclas para los Reales Bajeles. Esta demandó parte importante de la producción de hierro forjado de Cantabria (Corbera Millán, 1999, p. 48; Palacio Ramos, 1999, p. 147). El abastecimiento dependió sobre todo de ferrones que tenían a cargo una o varias "oficinas" (fábricas de anclas) privadas. Allí se reducía el mineral de la zona y se forjaban los tochos con los que se hacían las anclas. En líneas generales, estas instalaciones consistían en dos fraguas, que permitían calentar de forma simultánea e independiente los tochos de hierro que debían unirse, un yunque y unas carboneras. En aquel tiempo, para forjar un ancla de 58 quintales (castellanos) hacían falta unos diez hombres (ocho martilladores y dos maestros) y 24 días (Carrión Arregui, 1995, pp. 205-207).

El centro geográfico de producción de anclas para la Armada durante el siglo XVIII y parte del XIX fuelos alrededores de San Sebastián, desde Aia y Orio, pasando por Usurbil y Hernani, hasta llegar a Rentería, Guipúzcoa, País Vasco. Esta industria tuvo su auge a partir de la década de 1750 , aunque hundía sus raíces en los conocimientos de especialistas que trabajaron en la zona desde el siglo XVI. La producción de grandes anclas en Guipúzcoa estuvo vinculada con el énfasis puesto por los Borbones en la reconstrucción de la Armada y en el desarrollo de las industrias locales, así como con la demanda del exterior, principalmente de Francia. El maestro ancorero Juan Fermín de Guilisasti, responsable de la fabricación de anclas para la Armada entre 1738 y 1750, cumplió un rol destacado. Hacia 1730 visitó Holanda, donde pudo tomar conocimiento de una serie de técnicas que luego habría introducido en su ferrería de Arrazubia, en Aia, para producir anclas de mayor porte. A partir de mediados del siglo XVIII, la Corona realizó varios asientos con los dueños de las ferrerías y oficinas de Hernani. La producción de la región decayó hacia 1790 con el descenso de la construcción naval, entre otros factores, decadencia que se vio acentuada luego de la Batalla de Trafalgar y con la pérdida de las colonias ultramarinas (Carrión Arregui, 1995, pp. 199-212, ver también las referencias sobre Guilisasti en la obra de Egaña, 1788, pp. 158, 159).

\section{Especificaciones sobre el proceso de forjado}

Forjar un ancla era una actividad compleja. En el siglo XVIII, la caña y los brazos se elaboraban por separado y luego se soldaban a golpe de martillo. Esta tarea dependía, fundamentalmente, de la experticia de los artesanos (Pering, 1819, p. 18). En aquella época no era posible obtener grandes lingotes o tochos de hierro, razón por la que las partes principalesde las anclas de mayor tamaño - con estas se equipaban los navíos y fragatas - se elaboraba a partir de varios segmentos de hierro que se calentaban y soldaban entre sí. En términos generales, así se trabajaba en las ancorerías de las potencias marítimas europeas. No obstante, según el método y las técnicas utilizadas para dar forma y unir las referidas partes, puede establecerse una distinción entre dos modalidades. 
En Inglaterra, Francia y Holanda, la caña y los brazos de las anclas se realizaban soldando atadillos de barras de hierro que tenían la longitud de la parte en cuestión. La cantidad de varillas de cada haz dependía del tamaño del ancla (ver Figura 8) ${ }^{14}$. Esta operación se realizaba en caliente, con martinetes accionados por medio de energía hidráulica o, si no había suministro suficiente de agua, de forma manual (Curryer, 1999, pp. 62-64). Las partes así obtenidas se calentaban nuevamente y se soldaban mediante martillado, como se ilustra en las láminas de La Enciclopedia. Los brazos se unían a la caña por medio de encastre y soldadura, que se lograba usando un martinete. Durante esta instancia, el espacio que quedaba entre ambas partes se cubría con tiras de hierro. La soldadura de las palmas a los brazos, así como otros procedimientos menores, se realizaba por medio de forja manual. Un último término, los operarios desbastaban a fuerza de brazo la superficie para eliminar imperfecciones y colocaban el cepo de madera.

Aunque la manufactura de anclas era una de las expresiones más cabales de la tecnología de forjado del hierro, el proceso adolecía de varios defectos: era extremadamente difícil lograr una completa unión entre los metales por medio del martillado, el calentamiento de las superficies de contacto no era uniforme, no existían procedimientos rigurosos para la detección de fallas internas (e. g. la unión parcial de las barras) y el hierro utilizado en numerosos casos era de regular calidad (Jobling, 1993, p. 87). Soldar apropiadamente las barras de hierro que daban forma a la caña y los brazos era una tarea ardua, incluso utilizando los martinetes hidráulicos. Además, para facilitar la unión de las barras, estas debían tener un perfil regular y estar bien escuadradas. Ello difícilmente podía lograrse en una ferrería típica, mientras que las fanderías, aunque contaban con el equipamiento necesario, no eran numerosas fuera de Gran Bretaña. Este inconveniente fue resaltado por el ancorero español Guilisasti en 1744 y por el inspector de la fábrica de anclas de Hernani, Francisco A. de Oquendo, que le transmitió una impresión semejante al Marqués de la Ensenada, en 1752 (Carrión Arregui, 1995, p. 203). Refiriéndose a la producción de anclas en Inglaterra, Francia y Holanda, Oquendo notó lo siguiente:

Este atadillo de barras van calentando hasta que lleguen a unirse todas ellas, aunque a la verdad será quasi inasequible el hazer bien perfectamente esta operación. Después le ponen por encima yaplas o planchas caldeadas [hechas huasca], y por este medio, como quedan cubiertas aquellas barras, es imposible de reconocer si están bien o mal unidas (Oquendo, 1752, en Carrión Arregui, 1995, pp. 203- 204).

En las oficinas de anclas de la región guipuzcoana se utilizaba un método más lento e intensivo con respecto a la mano de obra, pero que requería poca inversión e infraestructura. El procedimiento consistía en soldar tochos cortos y gruesos, que se calentaban por separado (uno en cada fragua) y unían a fuerza de martillo, sin energía hidráulica, hasta lograr formar el largo de la caña o los brazos. Así, era más sencillo verificar que la soldadura de los fierros estuviera bien hecha. La introducción del carbón mineral (coque) por parte de Guilisasti fue la clave que permitió lograr una soldadura adecuada para fabricar las grandes piezas con que se equiparon los barcos de la Real Armada española. En cuanto al equipo necesario, el obrador que tenía este último en Aia contaba con un mazo de unas 18 a 20 arrobas, i. e. ca. $200 \mathrm{~kg}$ (Carrión Arregui, 1995 , pp. 202, 204, 205; 1998, pp. 552, 553) ${ }^{15}$.

Por semejante motivo que en el caso de las barras, la correcta unión de las partes de un ancla era un aspecto fundamental. Las exigencias mecánicas que operaban sobre estos elementos durante el uso podían ocasionar su rotura. Por ejemplo, durante las maniobras con el cabrestante para suspender el ancla del fondo, si esta se encontraba fuertemente agarrada, podía "arrancarse de patillas", expresión que refería a la rotura de uno de sus brazos (O'Scanlan, 1831, p. 220). Los sectores que estaban más comprometidos eran: cerca de la sección media de la caña; en la parte media de los
14. La cantidad de segmentos liados guardaba relación con el tamaño de cada una de las partes del ancla, así como con su porte. Steel señaló que la elección del número de barras necesarias estaba regulada por la experiencia (Steel, 1794, p. 78).

15. Las referencias acerca de las dimensiones de los mazos utilizados en las usinas metalúrgicas del siglo XVIII son escuetas. Martínez y Bohigas Roldán (1999) estimaron que la cabeza del mazo de la ferrería de Cades, en Herrerías, pudo pesar unos $500 \mathrm{~kg}$ y tener una cadencia entre 80 y 150 golpes por minuto. 
16. Light (2000) resaltó la importancia que tiene el conocimiento del trabajo de conformado del hierro forjado para el análisis de objetos históricos. Las fibras que presenta este material bajo ciertas condiciones, que adoptan una apariencia similar a las vetas de la madera, constituyen evidencia singular para estudiar su fabricación. brazos, a la altura de la base de las palmas y en el ángulo que formaban estos últimos con aquella. Al levar un ancla, la caña actuaba como una palanca y forzaba al brazo a desprenderse del fondo. De existir alguna debilidad en la unión de los brazos con la caña, debido a un empalme defectuoso, el primero podía quebrarse fácilmente. Asimismo, si la soldadura entre el brazo y la palma no era completa, esta podía ceder y perderse, o bien el brazo podía quebrarse a la mitad de su longitud. Por otro lado, si la caña era muy esbelta, podía no soportar el esfuerzo para desaferrar el brazo y, de resultas, doblarse y/o romperse. Conscientes de ello, los ancoreros procuraban tener especial cuidado en la manufactura y reforzar los sectores más comprometidos, e.g. en la cruz, procurando que no quedaran imperfecciones (Jobling, 1993, pp. 81, 84). En esta zona, la caña terminaba en una espiga que servía para empalmar los brazos, en cuya base también tenían un rebaje, de uno y otro lado. La forma de la junta resultante podía ser en bisel (junta oblicua) o a tope (junta recta).

La información histórica y arqueológica sugiere que estas variantes responden al modus operandi de diferentes regiones. Con respecto a la primera, el caso del ancla que perteneció al HMB Endeavour (1764-1778) una vez más es ilustrativo. La observación macroscópica y radiográfica de la pieza permitió reconstruir el proceso de empalme de los brazos a la caña. El tipo de junta utilizado, en bisel, dejaba un espacio entre la lengüeta de la caña y cada uno de los brazos, que era rellenado con trozos de hierro. Debido a las limitaciones propias de la forma de los martinetes, es probable que esta última operación fuese realizada manualmente. Aunque se emplearon dos planchas de refuerzo a cada lado de la unión, quedaron algunas cavidades internas. El análisis radiográfico indicó, además, que en algunos sectores del ancla las barras utilizadas no estaban bien soldadas entre sí (Samuels, 1992, pp. 81-86). Al respecto, puede citarse el caso de un ancla localizada en Puerto Deseado, Santa Cruz, Argentina, que probablemente perteneció a la corbeta de guerra HMS Swift (1763-1770). Esta pieza presenta indicios de un proceso de forja de similares características al empleado para fabricar el ejemplar del Endeavour (ver Ciarlo et al., 2011). En estos dos casos, debido al proceso corrosivo que afectó al material a nivel superficial, se reconoció a ojo desnudo el modo en que se superponen los brazos a la caña y el sector con material de relleno (Figura 9) ${ }^{16}$.

En las láminas 8 y 10 de la referida sección de La Enciclopedia se ilustran la forma de la caña y los brazos y el modo en que están unidos. A diferencia de las anclas británicas descriptas, en las francesas la lengüeta de la caña y el rebaje de los brazos eran rectos. Aun cuando este tipo de encaje (a tope) suponía un contacto más pleno entre la superficie de las partes, a ambos lados del ancla quedaban espacios vacíos. Estas hendeduras también eran tapadas, a fin de lograr una adecuada soldadura de las partes.

Existen ejemplares de anclas europeas de numerosos sitios arqueológicos del período localizados alrededor del globo, aunque las descripciones suelen ser sucintas. Varios trabajos de carácter general reportan sus características morfológicas y dimensiones principales (Breen y Forsythe, 2007; Cowan, Cowan y Marsden, 1975; Gesner, 1998; Nash, 2001; Rodgers et al., 2006; Smith, 2000; Stanbury, 1994; von Arnim, 1998; entre otros). Estas piezas constituyen una fuente de información de singular importancia para el estudio de la tecnología de la época, pasible de ser mejor explorada. En este sentido, los estudios de caracterización de materiales resultan de utilidad para determinar la calidad del hierro y los métodos empleados para su fabricación (Ciarlo et al., 2011; Deacon, 2007; Reyes et al., 2010; Samuels, 1992; Svoboda, De Rosa y Lorusso, 2005).

\section{De la calidad de las anclas para el servicio naval}

Las anclas podían presentar notables diferencias de calidad según el lugar adonde se producían y el material utilizado. Al respecto, dadas las consecuencias nefastas asociadas a la ineficacia de los elementos de fondeo, Blackburn enfatizó que todas 

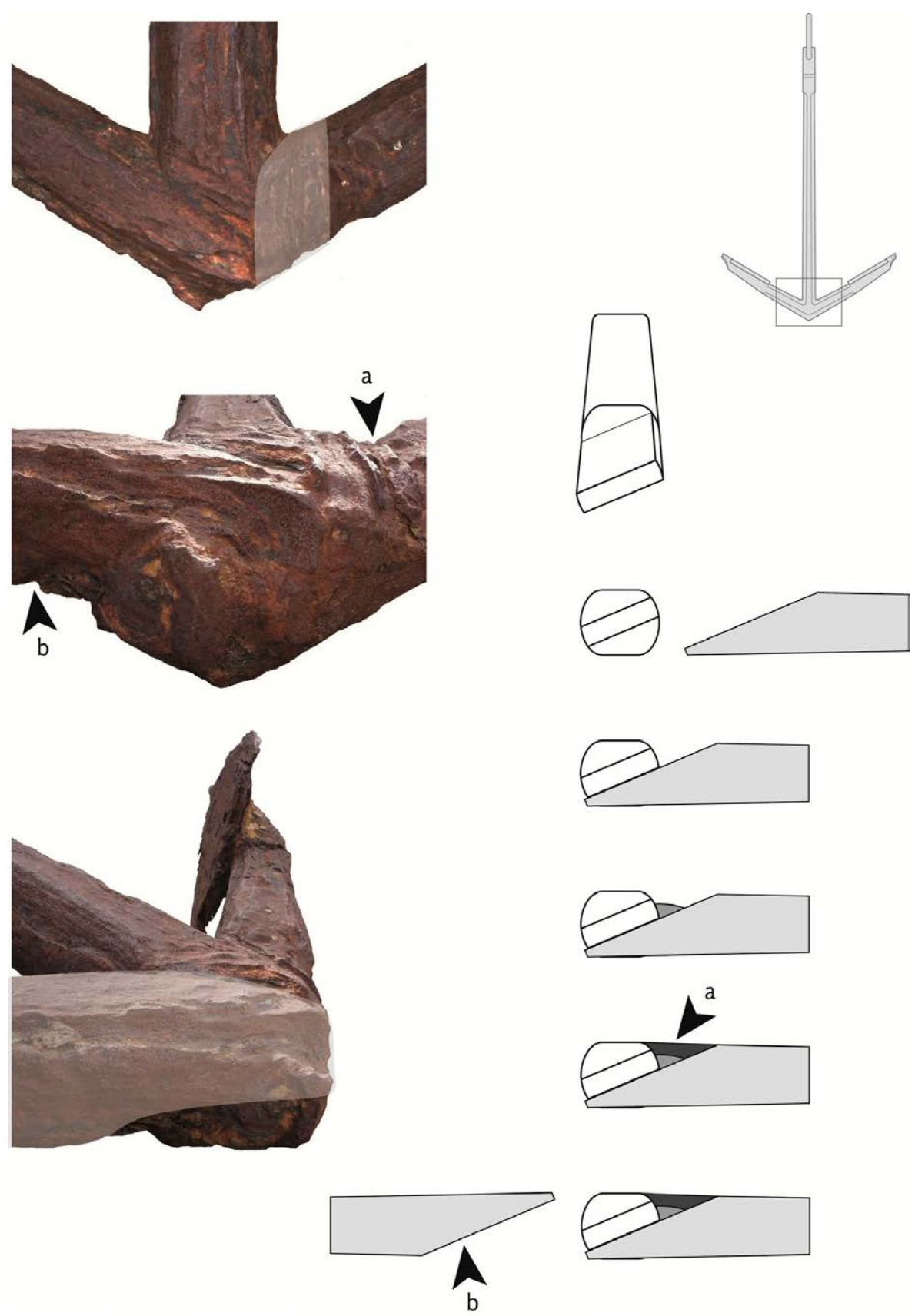

Figura 9. Detalles del ancla hallada en inmediaciones del sitio Swift y diagrama del empalme en bisel. Las flechas indican: la zona de refuerzo (a) y el bisel de uno de los brazos (b). Estos rasgos también están resaltados en las figuras de la izquierda (Gráfico: N. Ciarlo, 2009).

las anclas de los barcos de guerra debían fabricarse con el mejor hierro que pudiera obtenerse (Blackburn, 1817, p. 183).

Las propiedades del hierro de Suecia eran bien conocidas por aquel entonces. Este metal y el cobre constituían la principal riqueza del reino, que contaba con abundante cantidad de minas. Desde allí se exportaba hierro en barras y láminas y manufacturas tales como cañones, municiones, anclas y clavos. Parte importante de la producción de hierro era absorbida por los británicos, insumo fundamental para su industria (Boy, 1840, p. 724; Kent, 1973, p. 59). El hierro más afamado era de Öregrund, fabricado en varios talleres de Roslagen con mineral del yacimiento de Dannemora, Uplan, al 
17. Rodger destacó que Cort fue el primer 'maestro del hierro' británico en venderle hierro forjado a la Armada (Rodger, 2006, p. 376).

18. Las pruebas realizadas en varios astilleros británicos demostraron que la resistencia y tenacidad del hierro pudelado eran similares, e incluso superiores, a las del importado de Suecia (Curryer, 1999, pp. 65-71). norte de Estocolmo. Los especialistas de la época consideraban que la superioridad de este mineral estaba relacionada con el contenido de manganeso (o silicio) asociado (Thomson, 1813, p. 191).

Por otro lado, ya en 1611 Tomé Cano había resaltado que los hierros de España o Nápoles eran muy suaves (dúctiles), lo que permitía que las anclas fueran largas y se aferrasen bien al fondo; no así los de Flandes, que eran agrios (frágiles) y por ende la caña de las piezas debía hacerse más gruesa y corta, a fin de evitar que se rompieran. Por ello, las anclas españolas no requerían ser tan pesadas y no se quebraban, o lo hacían menos, con el uso (Carrión Arregui, 1995, p. 200). En su memoria sobre la provincia de Guipúzcoa, Bernabé A. de Egaña describió al hierro extranjero como "mal sobado, peor forjado, vidrioso, agrio y saltadizo" y resaltó que "el clavo para ser bueno ha de doblarse antes, que quebrárse” (Egaña, 1788, p. 223). Los ancoreros de las fábricas de Guipúzcoa utilizaban mineral de las minas de Somorrostro, en la provincia vecina de Vizcaya, que era considerado de la mejor calidad.

En Inglaterra, en cambio, el hierro producido localmente contenía un alto grado de impurezas. Asimismo, el coque utilizado como combustible durante el siglo XVIII atentó contra la calidad de los productos. La presencia de un elevado porcentaje de azufre y bajo contenido de manganeso en el hierro, lo tornaba frágil al ser trabajado a alta temperatura. Este estado era conocido como red o hot-short. Por otro lado, el fósforo surtía un efecto nocivo similar, pero a bajas temperaturas, denominado cold-short. Este comportamiento fue destacado recién en 1783 por el químico sueco Bergman, si bien los herreros especializados conocían por experiencia las cualidades del hierro extranjero desde larga data. Por tales razones, el Almirantazgo británico requirió que en los arsenales navales se empleara únicamente la materia prima procedente de Suecia o España (Samuels, 1992, pp. 86-88). Puntualmente, el referido producto de Öregrund fue considerado esencial (Evans, Jackson y Rydén, 2002, p. 652).

Recién a partir de finales del siglo XVIII, y sobre todo durante la siguiente centuria, fue mejorando la calidad de las anclas. Contribuyeron en este proceso el método de pudelado - por el que Henry Cort obtuvo una patente en $1783^{17}$ - y los subsiguientes adelantos técnicos en siderurgia, $e . g$. el uso de martinetes accionados con máquinas de vapor, hacia la década de $1840^{18}$. Pocos cambios ocurrieron hasta que se logró adelantar en la tecnología de forjado del hierro. Es decir, las mejoras introducidas en las anclas dependieron estrechamente del avance en los conocimientos, habilidades y equipamiento relacionados con el proceso de forja (Jobling, 1993, p. 121).

Resta mencionar que, desde temprano, Sutherland llamó la atención sobre la utilidad que tendría una maquinaria para testear las anclas antes de ser puestas en servicio. No obstante, las primeras máquinas de este tipo empleadas por el gobierno británico datan de 1833 (Curryer, 1999, p. 151). Durante el siglo XVIII, más allá de una revisión visual elemental no existía un sistema para comprobar la calidad de los productos (Jobling, 1993, p. 84). Un método indirecto para chequear la soldadura de las partes de un ancla consistía en golpearlas con un fierro y escuchar el sonido. Oquendo, haciendo referencia a la extraordinaria calidad de las anclas producidas por Guilisasti, resaltó que estas sonaban como campanas (Carrión Arregui, 1998, p. 553).

\section{Recapitulando}

Las anclas de hierro con cepo de madera eran un elemento fundamental del equipamiento de los barcos de guerra modernos. A lo largo de los siglos XVII y XVIII, la tendencia fue emplear un menor número de piezas, cada vez más pesadas. En esta última centuria, y por primera vez, se prestó especial atención a las características de 
los modelos, que quedaron plasmados en tratados o apartados realizados por técnicos y académicos. El modelo británico Old Admiralty Longshank fue distintivo y mostró notable regularidad desde su implementación. Las piezas francesas y españolas, por otro lado, exhibían similitudes entre sí en su aspecto exterior. En general, la forma y el ángulo de los brazos fueron establecidos desde temprano por especialistas que encontraron una solución de compromiso entre la capacidad de agarre y fortaleza (i. e. resistencia a la tracción) de las piezas. De allí que, aun cuando pueden notarse ligeras modificaciones, las anclas del período no experimentaran sustanciales cambios morfológicos. Los trabajos y ordenanzas de la época reportan información tabulada sobre las dimensiones de las anclas, que junto con las pautas de diseño constituyen una fuente relevante para identificar los ejemplares arqueológicos. Allende los aspectos diagnósticos ya referidos, solían existir leves diferencias en la forma y el porte de las anclas de un determinado lugar. En este sentido, no puede hablarse de una estandarización propiamente dicha, máxime cuando su producción dependía de los criterios marcados por la experiencia de cada maestro ancorero. Lo anterior pone de manifiesto, además, la distancia que existía entre la teoría - lo normativo, en este caso-y la práctica.

La manufactura de estas piezas puede considerarse una de las actividades metalúrgicas más complejas de su tiempo. El proceso de forjado solía realizarse en usinas especiales e incluía una combinación de operaciones manuales, que requerían de cierto equipamiento mecánico, e. g. martinetes y grúas. Debido a las limitaciones técnicas para producir grandes masas de hierro, la caña y los brazos de las anclas debían elaborarse uniendo varias barras de hierro. El mayor desafío residía en lograr una adecuada unión entre las superficies, ya que una soldadura imperfecta podía tener consecuencias aciagas. El método empleado en Gran Bretaña y Francia, básicamente, era similar. Cada una de las partes se obtenía a partir de un haz de barras que se forjaba hasta obtener una masa sólida, i. e. la caña y los brazos. En España, en lugar de los atadillos, los martilladores soldaban entre sí tochos cortos, que tenían la sección de la caña yde los brazos. Mediante este sistema podía trabajarse a una temperatura uniforme, que permitía la completa unión de las superficies de contacto. De allí que las anclas de la península, puntualmente las fabricadas en el norte fueran tan apreciadas.

La calidad de la materia prima también fue una faceta esencial. Las menas de hierro de España eran consideradas de excelencia. En Inglaterra, en cambio, los minerales tenían gran contenido de azufre y fósforo, que se sumaban a las impurezas perjudiciales del coque usado como combustible. Por ello, esta potencia marítima debió importar grandes volúmenes de hierro forjado durante el siglo XVIII, fundamentalmente de Suecia. Hacia el último cuarto de la centuria, se introdujo el método de pudelado, que mejoró la tecnología del hierro forjado y, por extensión, afectó a diversas industrias navales. Con relación al estudio de la fabricación, los análisis arqueométricos han permitido ahondar en el conocimiento de aspectos técnicos tales como la calidad de las barras de hierro, la forma de unión de los componentes y el control de la temperatura durante el proceso. Hasta la fecha, los ejemplares estudiados corresponden a anclas británicas de la segunda mitad del siglo XVIII, que muestran notables semejanzas entre sí. Cabe notar, también, que este tipo de análisis constituye una vía complementaria para evaluar la procedencia de las piezas.

Hubo que esperar hasta la siguiente centuria, luego de las Guerras Napoleónicas, para vislumbrar los siguientes cambios significativos en la forma y el material de las anclas. Destacan la introducción de nuevos diseños, con cepo metálico y cadena de hierro. Estas innovaciones dependieron de mejoras previas en torno a la calidad de la materia prima y la tecnología de forja, así como de nuevos conceptos en materia de diseño. En el caso de la Real Armada británica, las novedades estuvieron sujetas a un extenso período de prueba. Esta situación, entre otras, ilustra los condicionamientos técnicos 
que subyacen a ciertos cambios, así como el conservadurismo frente a la incorporación de propuestas que se apartaban de los usos de larga data.

\section{Agradecimientos}

A Amaru Argüeso y a los dos revisores externos, por sus valiosos comentarios, que enriquecieron el contenido del escrito original. Este artículo fue desarrollado a partir de la información expuesta en un capítulo de la tesis doctoral del autor, que trata sobre el conflicto naval y las innovaciones tecnológicas de los barcos de guerra europeos entre $c a .1750$ y 1815. 


\section{Q Referencias citadas}

"Blackburn, I. (1817). A Treatise on the Science of Ship-Building; with observations on the British Navy. Londres: James Asperne.

" Blanckley, T. R. (1750). A naval expositor. Londres: E. Owen.

》Bougainville, L. A., de (1771). Voyage autour du monde par la frégate du roi «la Boudeuse» et la flûte «''Étoile»; en 1766, 1767, 1768 \& 1769. 1771. París: Le Breton.

» Bouguer, P. (1746). Traité du Navire, de sa Construction, et de ses mouvements. París: C. A. Jombert.

» Boy, D. J. (1840). Diccionario Teórico, Práctico, Histórico y Geográfico de Comercio (Tomo 4). Barcelona: Imprenta de Valentín Torras.

» Brack, H. G. (Ed.). (2008). Handbook for Ironmongers: A Glossary of Ferrous Metallurgy Terms. Museum Publication Series, Hand Tools in History (Volumen 11), The Davistown Museum. Maine: Pennywheel Press.

» Breen, C. y Forsythe, W.(2007). The French Shipwreck La Surveillante, Lost in Bantry Bay, Ireland, in 1797. Historical Archaeology, 41(3), 39-50.

"Carrión Arregui, I. M. (1995).Tecnología tradicional y desarrollo de una industria pesada: la fabricación de anclas en Guipúzcoa (siglos XVI-XVIII). Revista de Historia Industrial, 7, 199-214.

》 Carrión Arregui, I. M. (1998). La fabricación de grandes anclas de navíos en el siglo XVIII en Gipuzkoa. Itsas Memoria. Revista de Estudios Marítimos del País Vasco, 2, 549-571.

"Chambaud, L. y Des Carrières, J.-T. H. (1815). A New Dictionary English and French, and French and English (Volumen 2, 5a. ed.). Londres: Cadell \& Davis.

" Ciarlo, N. C., De Rosa, H., Elkin, D., Svoboda, H., Vázquez, C., Vainstub, D. y Diaz Perdiguero, L. (2011). Examination of an 18th century English anchor from Puerto Deseado (Santa Cruz Province, Argentina). Historical Metallurgy, 45(1), 17-25.

»Corbera Millán, M. (1999). Ferrerías en Cantabria. En AA.VV., Hierro al mar (pp. 45-51). Cantabria: Instituto de Estudios Cántabros y Asociación Tajamar.

» Cotsell, G. (1856).Treatise on ship's anchors. Londres: J. Weale.

》 Cowan, R., Cowan, Z. y Marsden, P.(1975). The Dutch East Indiaman Hollandia wrecked on the Isles of Scilly in 1743. The International Journal of Nautical Archaeology and Underwater Exploration, 4(2), 267-300.

» Curryer, B. N. (1999). Anchors. An Illustrated History. Londres: Chathan Publication.

"Deacon, R. (2007). Analysis of 19th Century Boat Anchor Using Field Metallography Techniques. Advanced Materials \& Processes, 165(2), 22-23.

»Diderot, D. y J. R. d'Alembert (Eds.). 1769. Recueil de planches sur les sciences, les arts libéraux, et les arts méchaniques, avec leur explication (Volumen 7). París: Briasson y Le Breton.

"Dufief, N. G. (1810). A New Universal and Pronouncing Dictionary of the French and English Languages (Volumen 3). Filadelfia: T \& G. Palmer.

"Dunmore, J. (Ed.). (1981). The expedition of the St. Jean-Baptiste to the Pacific, 1769-1770: from journals of Jean de Surville and Guillaume Labé. Londres: Hakluyt Society. 
" Duyker, E. (Ed.). (1992). The discovery of Tasmania: journal extracts from the expeditions of Abel Janszoon Tasman and Marc-Joseph Marion Dufresne, 1642 \& 1772. Hobart: St. David's Park Publishing, Tasmanian Government Printing Office.

»Egaña, B. A. de (1788).Continuación de la memoria, que sobre las fabricas de anclas, de palanquetas, de baterias de fierro, la fanderia y otros establecimientos de la Provincia de Guipuzcoa, diò à lùz Don Juan Antonio Enriquez. Tolosa: Don Francisco de La Lama.

»Evans, C., Jackson, O. y Rydén, G. (2002). Baltic iron and the British iron industry in the eighteenth century. The Economic History Review, New Series, 55(4), 642-665.

» Falconer, W. (1780). An universal dictionary of the marine. Londres: T. Cadell.

" Gay, J. (1997). Six millénaires d'histoire des ancres. París: Presses de l'Université de ParisSorbonne.

» Gesner, P. (1998).Managing Pandora's Box: an exercise in eco-archaeometry. En M. Bound (Ed.), Excavating Ships of War (pp. 230-235). The International Maritime Archaeology Series (Volumen 2, University of Oxford). Shropshire: Anthony Nelson Publishers.

" Jobling, H. J. (1993).The History and Development of English Anchors ca. 1550 to 1850. (Tesis de Maestría inédita), Texas A\&M University, College Station, EE.UU.

» Kent, H. S. K. (1973).War and Trade in Northern Seas. Anglo-Scandinavian economic relations in the mid-eighteenth century. Cambridge: Cambridge University Press.

» Knuckey, P. J. (1988). An Anchor from HMB Endeavour: Restoration and other Aspects. Melbourne: Materials Research Laboratory.

» Light, J. D. (2000). Observations concerning the hand forging of wrought iron. Materials Characterization, 45, 327-340.

»López Martín, S. J. (2017). Inventario de los elementos de hierro y otros metales en los navíos españoles de 70 cañones. (Trabajo de fin de Máster inédito), Facultad de Filosofía y Letras, Universidad de Cádiz, España.

"Lusardi, W. R. (2000). The Beaufort Inlet shipwreck project. The International Journal of Nautical Archaeology, 29(1), 57-68.

» Martínez, J. M. y Bohigas Roldán, R.(1999). Excavación arqueológica de la ferrería de Cades. En AA.VV., Hierro al mar (pp. 52-63). Cantabria: Instituto de Estudios Cántabros y Asociación Tajamar.

» Moore, L. L. (1801). The British mariner's vocabulary. Londres: Hurst.

» Nash, M. (2001).The Sydney Cove historic shipwreck (1797). En M. Staniforth y M. Hyde (Eds.), Maritime Archaeology in Australia: A Reader (pp. 108-110). Australia Meridional: Blackwood.

» O’Scanlan, T. (1831). Diccionario marítimo español. Madrid: Imprenta Real.

»Palacio Ramos, R. (1999). Transformación y comercio del hierro en la Bahía de Santoña y el Río Asón. En AA.VV., Hierro al mar (pp. 144-151). Cantabria: Instituto de Estudios Cántabros y Asociación Tajamar.

" Pering, R. (1819). A Treatise on the Anchor. Londres: Congdon \& Hearle.

"Pinault-Sørensen, M. (1992). Les métamorphoses des Planches: quelques exemples. Recherches sur Diderot et sur l'Encyclopédie, 12, 99-112.

» Réaumur, R.-A. F., y Duhamel du Monceau, H.-L. de [1764] (1993). Fabrique des Ancres. Dossier ANCRE (Volumen 1). San Remo: Pharos.

» Reyes, J., González-Sánchez, J., Barba, H., Huitz, C., Bernés, F., Escamilla, R. y Pérez, T. (2010). Estudios sobre Degradación del Patrimonio Histórico en el Estado de Campeche. Campeche: Universidad Autónoma de Campeche. 
» Rodger, N. A. M. (2006). The Command of the Ocean. A Naval History of Britain, 1649-1815. Londres: Penguin Books.

» Rodgers, B. A., Cantelas, H., Bowdoin, J., Hayes, R. L., Hermley, L. y Lentz, L. (2006). The 1996 Anguilla Shipwreck Survey (Research Report No. 15, Program of Maritime Studies). Greenville: East Carolina University.

» Rodríguez Mariscal, N. (Comp.). (2010). Sondeos arqueológicos subacuáticos en el pecio de Camposoto (San Fernando, Cádiz). Memoria final del Centro de Arqueología Subacuática del Instituto Andaluz de Patrimonio Histórico, Cádiz. Manuscrito inédito.

»Samuels, L. E. (1992). Australia's Contribution to Archaeometallurgy. Materials Characterization, 29, 69-109.

» Schwartz, F. J. y Green, J. (1962). Found: One anchor from HMS Dictator. Maryland Historical Magazine, 57(4), 367-370.

»Smith, T. (200o). Old Pattern Admiralty Long Shanked Anchor (North Head, Sydney). Conservation Management Plan. Sydney: NSW Heritage Office.

»Stanbury, M. (1994). HMS Sirius 1790: an illustrated catalogue of artefacts recovered from the wreck site at Norfolk Island (Special Publication No.7). Adelaide: Australian Institute for Maritime Archaeology.

»Steel, D. (1794). The Elements and Practice of Rigging and Seamanship. Londres: D. Steel.

» Sutherland, W. (1717). Britain’s Glory: or, Ship-Building Unvail'd. Londres: T. Norris.

»Svoboda, H. G., De Rosa, H. y Lorusso, H. (2005). Estudio de un ancla antigua hallada en el lecho del río de la Plata. Actas del Congreso Binacional SAM-CONAMET. Mar del Plata, Buenos Aires.

» Taillemite, E. (2003). Histoire ignorée de la marine française. París: Perrin.

» Tarlton, K. (1977). The search for and discovery of anchors lost in 1769 by the French explorer de Surville at Doubtless Bay, New Zealand. The International Journal of Nautical Archaeologyand Underwater Exploration, 6(1), 64-70.

» Telechea Idígoras, J. I. (1977). Anclas de Hernani: el registro de cartas de D. Francisco Antonio de Oquendo, Inspector de la Fábrica de Anclas (1750-1755). San Sebastián: Grupo Dr. Camino de Historia Donostiarra de la Real Sociedad Vascongada de los Amigos del País.

» Thomson, T. (1813). Travels in Sweden, during the autumn of 1812. Londres: R. Baldwin.

»Upham, N. E. (1983). Anchors. Londres: Shire Publications.

»Von Arnim, Y. (1998). The wreck of the 5th rated British frigate HMS Sirius (1797) in Mauritius. The Bulletin of the Australian Institute for Maritime Archaeology, 22, 35-44. 